\title{
Jiao-Tai-Wan Improves Cognitive Dysfunctions through Cholinergic Pathway in Scopolamine-Treated Mice
}

\author{
Xin-Chen Wang $\mathbb{D}^{1},{ }^{1}$ Yu-Min Xu, ${ }^{2}$ Hong-Ying Li, ${ }^{2}$ Chun-Ying Wu, ${ }^{1}$ Ting-Ting Xu, \\ Na-Chuan Luo, ${ }^{2}$ Shi-Jie Zhang $\mathbb{D}^{2},{ }^{2}$ Qi Wang $\mathbb{D}^{2},{ }^{2}$ and Shi-Jian Quan $\mathbb{D}^{1}$ \\ ${ }^{1}$ School of Pharmaceutical Sciences, Guangzhou University of Chinese Medicine, Guangzhou 510006, China \\ ${ }^{2}$ Institute of Clinical Pharmacology, Guangzhou University of Chinese Medicine, Guangzhou 510405, China
}

Correspondence should be addressed to Shi-Jie Zhang; zsj19891122@gmail.com, Qi Wang; wangqi@gzucm.edu.cn, and Shi-Jian Quan; quansj@gzucm.edu.cn

Received 21 March 2018; Accepted 9 May 2018; Published 27 June 2018

Academic Editor: Stavros Baloyannis

Copyright (c) 2018 Xin-Chen Wang et al. This is an open access article distributed under the Creative Commons Attribution License, which permits unrestricted use, distribution, and reproduction in any medium, provided the original work is properly cited.

\begin{abstract}
Cognitive dysfunction is characterized as the gradual loss of learning ability and cognitive function, as well as memory impairment. Jiao-tai-wan (JTW), a Chinese medicine prescription including Coptis chinensis and cinnamon, is mainly used for the treatment of insomnia, while the effect of JTW in improving cognitive function has not been reported. In this study, we employed a scopolamine(SCOP-) treated learning and memory deficit model to explore whether JTW could alleviate cognitive dysfunction. In behavioral experiments, Morris water maze, Y-maze, fearing condition test, and novel object discrimination test were conducted. Results showed that oral administration of JTW $(2.1 \mathrm{~g} / \mathrm{kg}, 4.2 \mathrm{~g} / \mathrm{kg}$, and $8.4 \mathrm{~g} / \mathrm{kg})$ can effectively promote the ability of spatial recognition, learning and memory, and the memory ability of fresh things of SCOP-treated mice. In addition, the activity of acetylcholinesterase (AChE) was effectively decreased; the activity of choline acetyltransferase (ChAT) and concentration of acetylcholine (Ach) were improved after JTW treatment in both hippocampus and cortex of SCOP-treated mice. JTW effectively ameliorated oxidative stress because of decreased the levels of malondialdehyde (MDA) and reactive oxygen species (ROS) and increased the activities of superoxide dismutase (SOD) and catalase (CAT) in hippocampus and cortex. Furthermore, JTW promotes the expressions of neurotrophic factors including postsynaptic density protein 95 (PSD95) and synaptophysin (SYN) and brain-derived neurotrophic factor (BDNF) in both hippocampus and cortex. Nissl's staining shows that the neuroprotective effect of JTW was very effective. To sum up, JTW might be a promising candidate for the treatment of cognitive dysfunction.
\end{abstract}

\section{Introduction}

Cognitive dysfunction (CD), a central neurodegenerative disease, is closely associated with cholinergic nerve impairment. CD is defined as deficiency in memory, speech, execution, spatial discrimination ability, etc., which may possibly progress to the Alzheimer's disease (AD) [1-3]. Cholinergic neuron plays an important role in learning and memory, whose survival depends on neurite outgrowth and nerve growth factor from the target-derived phenotypic differentiation [4-7]. Another study found that positron emission tomography (PET) data show that cognitive dysfunction as the clinical stage before the onset of dementia symptoms, with the performance of cholinergic dysfunction [8]. Those opinions support the hypothesis that $\mathrm{CD}$ is probably an early-stage of $\mathrm{AD}$, with the cholinergic nervous system affected simultaneously. Therefore, the treatment of cholinergic nervous system can alleviate the symptoms of $\mathrm{CD}$ and improve life quality of patients. Fujiwara et al. transplanted human choline acetyltransferase- (ChAT-) positive cholinergic neurons into the cortex of mice, which consequently alleviated the symptoms of $\mathrm{CD}$ by improving the cholinergic nervous system [9]. Acetylcholinesterase (AChE) inhibitors are used to treat encephalopathy by alleviating the symptoms of $\mathrm{CD}$ due to carbon monoxide poisoning [10]. Previous studies have confirmed that human neural stem cells overexpressing acetylcholine transferase (ChAT) play a role in the recovery of cognitive function in the rat model [11]. The medication of CD generally includes AChE inhibitors, donepezil, galantamine, $\mathrm{N}$-methyl-d-aspartate receptor antagonist, and 
tacrine, all of which are only capable of delaying the decline of cognitive function to some extent, rather than fundamentally change the progression of dementia.

Traditional Chinese medicines (TCMs) have a long history of research, continuously inherited and developed in the period of innovation. TCMs are a combination of several different active substances, which have the advantage of low toxicity and few side effects, leading to the wide application in the study of brain disease $[12,13]$. Jiao-tai-wan (JTW), a well-known prescription, was first mentioned in Han's Book on Medicine compiled by MaoHan. The main medical effect of JTW is restoration of normal harmony between heart and kidney, resulting in frequent applications in the treatment of insomnia. JTW contains two types of Chinese traditional medicines: Coptis chinensis (CC) and cinnamon (CIN). The main active ingredients of $\mathrm{CC}$ and CIN reduce neurological inflammation and alleviate the symptoms of CD [14-17]. Berberine is a mixture of alkaloids extracted from CC, which has a significant effect on relieving the symptoms of CD [18]. Cinnamic aldehyde, a major active constituent derived from CIN, is distinguished with anti-inflammatory, antioxidative, and neuroprotective effect [19]. In summary, we hypothesized that JTW is effective in neuroprotection.

In the present study, we investigated the effect of JTW and probed its potential mechanisms. Three different doses $(2.1 \mathrm{~g} / \mathrm{kg}, 4.2 \mathrm{~g} / \mathrm{kg}$, and $8.4 \mathrm{~g} / \mathrm{kg})$ of JTW were used to treat the scopolamine- (SCOP-) induced cognitive dysfunction in Kunming mice. Our study found that JTW could significantly alleviate cognitive impairment by improving central cholinergic neurotransmission and prevent SCOP-induced neurodegeneration of the cortex and hippocampus.

\section{Materials and Methods}

2.1. Animal and Treatment. Male Kunming mice weighed 21-25g were purchased from Guangzhou University of Traditional Chinese Medicine Experimental Animal Center (Guangzhou, China). The mice were kept in the cage for one week to adapt to the environment and were fed with free food and water. The animals were maintained at 22 $\pm 2^{\circ} \mathrm{C}$ temperature, 12 -hour light/12-hour dark cycle, and $60 \%$ relative humidity throughout the study. The animals were kept in accordance with Guiding Principles for the Care and Use of Laboratory Animals, which was adopted and promulgated by the United States National Institutes of Health. Mice were randomly assigned to six treatment groups ( $\mathrm{n}=10$ per group): control group was given $0.9 \%$ saline (CON, $\mathrm{n}=10$ ), scopolamine model group (SCOP, $\mathrm{n}=10$ ), donepezil group was supplied SCOP $3 \mathrm{mg} / \mathrm{kg}+$ ARI $3 \mathrm{mg}$ / $\mathrm{kg}(\mathrm{DON}, \mathrm{n}=10)$, the low dose has SCOP $3 \mathrm{mg} / \mathrm{kg}+$ JTW $2.1 \mathrm{~g} / \mathrm{kg}(J T W L, \mathrm{n}=10)$, the middle dose contains SCOP 3 $\mathrm{mg} / \mathrm{kg} \mathrm{Kg}+\mathrm{JTW} 4.2 \mathrm{~g} / \mathrm{kg}(\mathrm{JTWM}, \mathrm{n}=10)$, and the high dose was provided SCOP $3 \mathrm{mg} / \mathrm{kg}+\mathrm{JTW} 8.4 \mathrm{~g} / \mathrm{kg}$ (JTWH, $\mathrm{n}=10$ ). Mice were given saline, JTW, donepezil, respectively, for treatment once a day for two weeks by gavage. Donepezil and JTW were given 30 minutes before scopolamine injection. All behavioral tests were performed 30 minutes after the injection of scopolamine. Donepezil and JTW were administered once per day for two weeks throughout the experiment, and scopolamine was given once per day until the end of experiment. All of the animal experiments were approved by the Animal Ethics Committee of Guangzhou University of Chinese Medicine, in accordance with the guide for the animal experiments, clinical studies, and biodiversity rights.

2.2. Materials. Scopolamine hydrobromide was dissolved in sterile saline $(0.9 \% \mathrm{NaCl})$ at a concentration of $0.8 \mathrm{mg} / \mathrm{ml}$. Donepezil was dissolved in distilled water at a concentration of $1 \mathrm{mg} / \mathrm{ml}$. Donepezil was purchased from Shandong Jinan Dexinjia Bio-technology Limited Company (Shandong, China). Scopolamine hydrobromide injection (Guangzhou Baiyun Mountain Mingxing Pharmaceutical Co., Ltd., Guangzhou, China) was purchased from Guangzhou Pharmaceuticals Corporation (Guangzhou, China). Kits used for detection of choline acetyltransferase (ChAT), acetylcholine (Ach), acetylcholinesterase (AChE), reactive oxygen species (ROS), malondialdehyde (MDA), superoxide dismutase (SOD), and catalase (CAT) were purchased from the Nanjing Jiancheng Bioengineering Institute (Nanjing, China). Anti- $\beta$-actin was purchased from Sigma-Aldrich. Secondary antibodies (horseradish peroxidase conjugated antirabbit IgG and anti-mouse IgG) were purchased from Cell Signaling Technology, Inc. Primary antibodies including anti-synaptophysin (SYN), anti-postsynaptic density 95 (PSD95), and anti-brain-derived neurotrophic factor (BDNF) were purchased from Abcam, Inc.

\subsection{Preparation of JTW. JTW, composed of CC and CIN} in proportion to 10: 1, were purchased from the First Affiliated Hospital Pharmacy Room of Guangzhou University of Traditional Chinese Medicine (Guangzhou, China), followed by extraction process carried out at the Laboratory of Pharmacology, Guangzhou University of Traditional Chinese Medicine. The JTW extraction steps were as follows: (a) CIN was immersed for two hours with eight volumes of water, boiled with the extraction of volatile oil equipment for six hours, so that the benzine could be mostly extracted. (b) The dregs and CC were soaked with six volumes of water for two hours, then boiled for five hours. (c) The extraction of liquid was given low temperature heating to get the concentrated liquid. (d) Finally, the volatile oil was mixed into the concentrated liquid.

2.4. Morris Water Maze Test. Using Morris water maze test to evaluate the spatial learning and memory ability was mentioned in previous studies [20]. The Morris Water Maze Animal Behavioral Analysis System consists of a black circulation pool (Diameter: $120 \mathrm{~cm}$; Height: $40 \mathrm{~cm}$ ), a black platform (Diameter: $10 \mathrm{~cm}$ ), and a recording system. The pool was divided into four quadrants (NE, SE, SW, NW), and different shapes of paper were pasted at the midpoint of the quadrant of the pool wall, which served as the marker for mice to find the platform. The escape platform was placed in the target quadrant center $2 \mathrm{~cm}$ below the water. Morris water maze behavior tests to detect the learning and memory 
abilities of mice were conducted in darkness; therefore curtains should be blocked around the pool. Mice were given a place navigation test for four consecutive days. For each daily trial, there were four sequential training trials, beginning with placing the animal in the water facing the wall of the pool with drop location changing for each trial randomly, followed by the record system starting to record the time. The escape latency was recorded at the end. The mouse would be guided to the platform by the trainer and remained there for $10 \mathrm{~s}$ if it failed to find the platform within $60 \mathrm{~s}$, whose escape latency would be consequently recorded as $60 \mathrm{~s}$. On the sixth day, the platform was removed and mice were allowed to swim freely in the pool for $60 \mathrm{~s}$. The time of crossing through the original platform position, the time spent in the target quadrant, and the swimming speed were surveyed, which indicated the degree of memory amalgamation.

2.5. Y-Maze. The spontaneous alternation of the Y-maze has been reported as an indicator of immediate working memory performance [21]. Y- maze as the horizontal labyrinth is composed of three arms $\left(40 \times 4.5 \times 12 \mathrm{~cm}, 120^{\circ}\right)$. The maze of floors and walls are made of opaque polyethylene plastic in a black environment. The mice were initially placed in an arm and then placed in the sequence (e.g., 123213, etc.), and the times of each mouse entering the other arms within 5 minutes were manually recorded. Successful alternation was defined as consecutive entries into a new arm before returning to the two previously visited arms. It is necessary to clean up the labyrinth thoroughly and eliminate odor and stains before each test. The maze task was carried out after treatment with scopolamine for 30 minutes. The alternation percentage was defined according to the formula: [(number of alternations)/(total number of arm entries -2$)] \times 100$ [22].

2.6. Novel Object Discrimination (NOD). The novel object discrimination (NOD) test is a method for learning and memory test, based on the principle that animals have instinct to explore new objects [23]. Experimental device was composed of rectangular black box $(60 \times 25 \times 25 \mathrm{~cm})$ and three objects named A, B, C, of which $\mathrm{A}$ was identical to $\mathrm{B}$, while the $\mathrm{C}$ object was completely different (shape, color) from the A and B. NOD test was divided into two stages: familiarity and testing. In the stage of familiarity, the mouse as well as A and B objects were placed in the box together, adapting for five minutes. After 24 hours for the second phase, object B was replaced with $\mathrm{C}$. The long-term memory recognition (LTMR) was evaluated by the different objects at the same area. The time which the mouse spent distinguishing novel and similar objects could be calculated using the identify index $(\mathrm{TNI})=(\mathrm{TN}-\mathrm{TF}) /(\mathrm{TN}+\mathrm{TF})[24$, 25]. After each test, the instruments and objects were washed with $70 \%$ ethanol to reduce the olfactory hints.

2.7. The Fear Conditioning Test (FCT). The parameters and processes of fear conditioning were conducted by referring to previous studies [26]. The equipment of fear conditioning test includes experimental chamber transparent acrylic room $(30 * 30 * 30 \mathrm{~cm})$, the bottom of the grille floor associated with the shock generator $(0.1 \sim 1.0 \mathrm{~mA}$ shock $)$, the sound generator (broadband or low frequency) connected with a computer. The floor should be cleaned with $70 \%$ ethanol before each test and the interior space was white. Additionally, white noise and electrical stimulation were needed during the test. The mouse were placed into the chamber and allowed to explore for $60 \mathrm{~s}$, while a $2 \mathrm{~Hz}$ pulsation was set $(80 \mathrm{~dB}, 3600 \mathrm{~Hz})$ for 60 seconds and a slight foot shock $(0.8 \mathrm{~mA}, 0.5 \mathrm{~s})$ was given immediately for three times. Consolidations of contextual and auditory fear memory were elucidated, including the number of mice harboring "freezing behavior" in the absence of electrical stimulation of the same environment after 24 hours, the times of occurrences of the behavior to assess the learning and memory. Freezing was defined as completely immobile posture except for those related to respiration.

2.8. Measurement of Ach Level and ChAT, AChE Activity. All mice were immediately decapitated under anesthesia at the end of the behavioral tests. Hippocampus and cortex were carefully examined from the brain. All the processes were carried out in ice. Tissue were stored at $-80^{\circ} \mathrm{C}$ quickly. The hippocampus and cortical tissue were homogenized with cold saline. The homogenate was centrifuged at $12,000 \mathrm{x} g$ for 10 minutes at $4^{\circ} \mathrm{C}$. Supernatant was collected and total protein concentration was determined using a bicinchoninic acid (BCA) protein assay kit (Nianjing Jiancheng Bioengineering Institute, Nanjing, China) for the assay of the AChE and ChAT activities as well as measuring the level of Ach. Universal Microplate Spectrophotometer (Bio-Rad, Hercules, CA, USA) was used to detect the Ach concentration and the activities of ChAT and AChE according to the manufacturer's instructions.

2.9. ROS Production. The hippocampus and cortical tissue were homogenized with cold saline. The homogenate was centrifuged at $12,000 \mathrm{x}$ g for 10 minutes at $4^{\circ} \mathrm{C}$. Supernatant was collected to detect the levels of ROS, which was measured by DCFH-DA as a redox sensitive fluorescent dye. DCFH was oxidized to strong green fluorescent substance DCF in the presence of ROS, which has a maximum peak at excitation wavelength of $502 \mathrm{~nm}$ and emission wave length of 530 and intensity is proportional to intracellular reactive oxygen species.

2.10. CAT, MDA, and SOD Assays. The hippocampus and cortical tissue were homogenized with cold saline. The homogenate was centrifuged at $12,000 \mathrm{x}$ g for 10 minutes at $4^{\circ} \mathrm{C}$. Supernatant was collected to detect the levels of MDA and the activity of SOD and CAT using Universal Microplate Spectrophotometer (Bio-Rad, Hercules, CA, USA) according to the manufacturer's instructions.

2.11. Western Blot Analysis. Brain tissues of hippocampus and cortex were homogenized and lysed in sample buffer (0.5 M Tris/HCl pH 6.8, 50\% glycerol, 10\% sodium dodecyl sulphate (SDS), 1: 100 inhibitor proteases, and phosphatases cocktail). The lysate was centrifuged at $12,000 \times \mathrm{g}$ for $10 \mathrm{~min}$ at $4^{\circ} \mathrm{C}$, mixed with 1: 4 loading buffer and denatured by 
boiling at $100^{\circ} \mathrm{C}$. The same amount of protein $(30 \mu \mathrm{g})$ was subjected to SDS-polyacrylamide gel electrophoresis (PAGE) analysis and transferred to PVDF membranes. Thereafter, the membrane was blocked in 5\% bovine serum albumin (BSA) that dissolved in Tris-buffered saline-Tween-20 (TBST) for $1 \mathrm{~h}$ at room temperature. The membranes containing the protein were incubated with anti-PSD95, anti-SYN, anti-BDNF, and anti- $\beta$-actin overnight at $4^{\circ} \mathrm{C}$. Then the membrane was incubated with horseradish peroxidase conjugated anti-rabbit or anti-mouse for $1 \mathrm{~h}$ at room temperature. Finally, the bands on the membrane were visualized using the superenhanced chemiluminescence reagent (ECL; Applygen Technologies Inc., Beijing, China).

2.12. Nissl's Staining. Brain paraffin sections were washed in xylene and rehydrated by a graded series of ethanol and double-distilled water. Then these parts were immersed in a Nissl's stain (Nanjing Institute of Bioengineering, Nanjing, China) at room temperature for 5-10 minutes. The slides were rinsed in double-distilled water and dehydrated through $70 \%$, $95 \%$, and $100 \%$ gradient alcohol, and removed in xylene. Images were analyzed using optical microscopy and LEICA QWin Plus (Leica Microsystems, Wetzlar, Germany).

2.13. Statistical Analysis. Experimental values were given as means \pm SEM. SPSS 19.0 statistical software (IBM, Endicott, NY) was evaluated to performed all statistical analysis. Twoway analysis of variance (ANOVA) was applied among the different groups to analyze differences in data for the biochemical parameters, followed by Dunnett's significant post hoc test for pairwise multiple comparisons. Differences were considered as statistically significant at $p<0.05$.

\section{Results}

3.1. HPLC Analysis of JTW. We took advantage of methanol as extract condition to ensure the effective components of JTW. Phenomenex Luna-C18 was indicated as chromatographic condition $(250 \mathrm{~mm} * 4.6 \mathrm{~mm}, 5 \mu \mathrm{m})$, detection wavelength, $300 \mathrm{~nm}$; flow rate, $1 \mathrm{~mL} / \mathrm{min}$. As shown in Figure 1(a), the peak area of JTW in HPLC maps has eight compounds which can be identified as magnoflorine (Peak 1), groenlandicine (Peak 2), coptisine (Peak 3), jatrorrhizine (Peak 4), columbamine (Peak 5), berberine (Peak 6), palmatine (Peak 7 ), and cinnamaldehyde (Peak 8), by which the berberine was quantified as contents of main composition. The compounds above are compared with reference compounds (in Figure 1(b)).

\subsection{JTW Improves Learning and Memory of SCOP-Treated} Mice. In order to investigate whether JTW could alleviate the SCOP-treated spatial-working, learning and memory impairment, four types of behavioral tests were conducted: Morris water maze test, Y-maze, novel object discrimination, and fear conditioning test. As shown in Figures 2(a) and 2(b), within five training days, the mice progressively reduced the time to find hidden platforms. Compared with the control group, the SCOP intraperitoneal injection group had an extremely long time to find a hidden platform. However, the preprotective effect of Jiao-tai-wan and donepezil led to significantly shortened escape latency in JTW low-dose, middle-dose, and high-dose group as well as donepezil group when compared to the SCOP group. On the sixth day, the experiment of estimating their spatial-working memory was conducted, the platform was removed and the mice were allowed to swim freely. A longer latency was observed in the SCOP group, with relatively less time stayed at the target quadrant and fewer times crossing the position of the removed platform ( $p<0.001$, Figures 2(c), 2(d), and 2(e)). In the JTW and the DON groups, the time for the mice to stay in the target quadrant and the number of crossing platforms were prominently increased. The swimming speed decreased remarkably in the SCOP group, but there was no significant difference among the control, JTW, and DON groups.

Then, the effect of JTW on the SCOP-induced deficit was assessed using the Y-maze test, a well-known method for working memory (Figures 3(a) and 3(b)). Entry arm speed in Y-maze test was shown in Figure 3(a); the entry speed in JTW-treated group was decreased than control group. A remarkable increase of spontaneous alternation index was observed in the vehicle control group, in contrast to the SCOP group in Figure 3(b) $(p<0.01, p<0.001)$. The spontaneous alternation index of the JTW (low-dose, middle-dose, highdose) and DON groups have significant improve compared to the SCOP group.

In order to evaluate the impact and improvement of JTW on behavioral disorders, the NOD was applied to determine the ability of mice to distinguish between complex setting and different novel objects. The exploration average speed in JTW (low-dose, middle-dose, high-dose) groups was decreased than control group in NOD. However, the percent of NOD time of mice to explore novel objects was significantly increased in JTW (low-dose, middle-dose, highdose) groups (Figures 3(c) and 3(d)).

The fear conditioning test (FCT) was applied to evaluates ability to remember fear based on the freezing behavior number of times in mice, differences were observed between the control and the SCOP group in both the contextual and the cued recall paradigms, with less freezing times presented in the SCOP group $(p<0.001$,). In contrast, the JTW and DON groups have more freezing times (Figures $4(\mathrm{a})$ and $4(\mathrm{~b}))$.

3.3. JTW Improves the Cholinergic Nerve System in SCOPTreated Mice. In order to illuminate the potential mechanism of JTW in ameliorating cognition deficiency in SCOP-mice, the activities of cholinergic marker enzymes were detected. In the group of JTW and DON, the level of acetylcholine (Ach) was significantly improved in both hippocampus and cortex. However, Ach level of SCOP group was evidently declined (Figures 5(a) and 5(d)). The acetylcholinesterase (AChE) activity was increased by scopolamine in the hippocampus and cortex $(p<0.001, p<0.01$, shown in Figures 5(b) and $5(\mathrm{e}))$, while it was significantly decreased by the treatment with JTW and DON groups. As shown in Figures 5(c) and 5(f), the activity of choline acetyltransferase (ChAT) were 


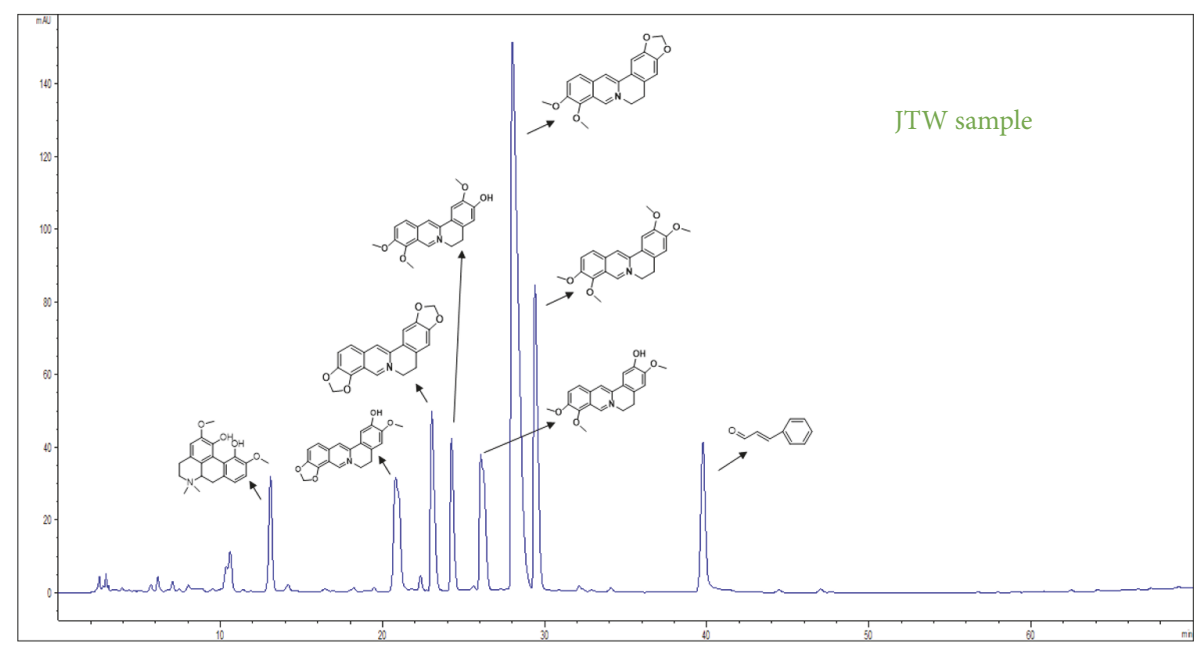

(a)

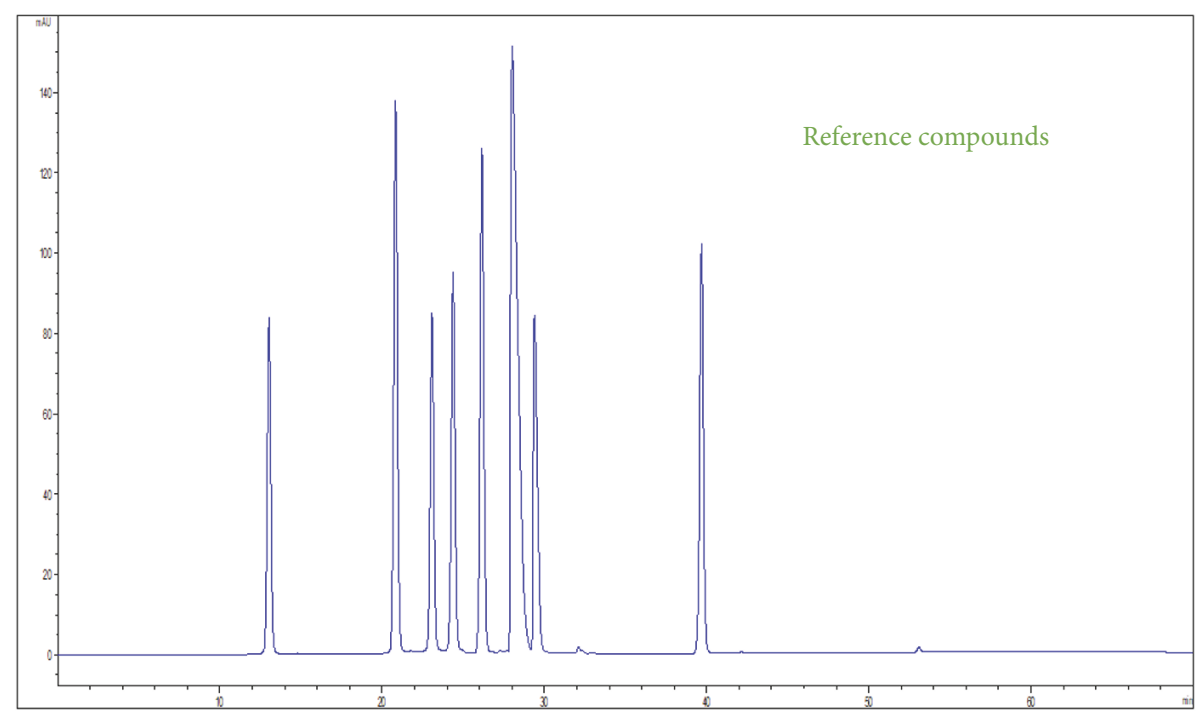

(b)

FIGURE 1: HPLC of JTW. HPLC profiles were applied to analyzed chemical standardization of JTW and reference compounds. (a) JTW samples; (b) reference compounds.

significantly increased with the application of JTW and DON, whereas they were sharply decreased in the SCOP-treated group in both hippocampus and cortex. In summary, JTW can protect cognitive deficits by affecting cholinergic nervous system.

3.4. JTW Improves the Oxidative Stress Status in SCOP-Treated Mice. Oxidative stress state can be used to determine the state of hippocampus and cortex of SCOP-treated mice. Scopolamine had a robust effect on in the hippocampus and cortex. Consequently, increased levels of MDA and ROS (Figures 6(a), 6(b), 6(e), and 6(f)), and suppressed activities of SOD and CAT (Figures 6(c), 6(d), 6(g), and 6(h)) were observed in the SCOP group, JTW and DON groups respectively. Obviously, the MDA and ROS levels were ameliorated by JTW and DON, which were able to increase the activity of SOD and CAT at the same time. In contrast, the SCOP group did not improve activities of SOD and CAT and levels of MDA and ROS.

3.5. JTW Improves the Neurodegeneration in SCOP-Treated Mice. As shown in Figures $7(\mathrm{a})$ and $7(\mathrm{~b})$, the expression of brain-derived neurotrophic factor (BDNF), postsynaptic density 95 (PSD95) and synaptophysin (SYN) as neurotrophic factors were sharply decreased in the SCOP-treated mice. Due to the treatment of JTW and DON, the protein returned to normal levels and were fully expressed. Nissl's staining was applied to hippocampus as a further nerve cell test method (Figure 7(c)). In the hippocampal subfield of SCOP-treated mice, the neurons were significantly shrunken, irregularly arranged, and weakly stained, which indicated that neurons were diffusely deteriorated or dead and great quantity Nissl bodies were lost in these neurons. Conversely, the neurons exhibited regularly arrange, normal morphology 


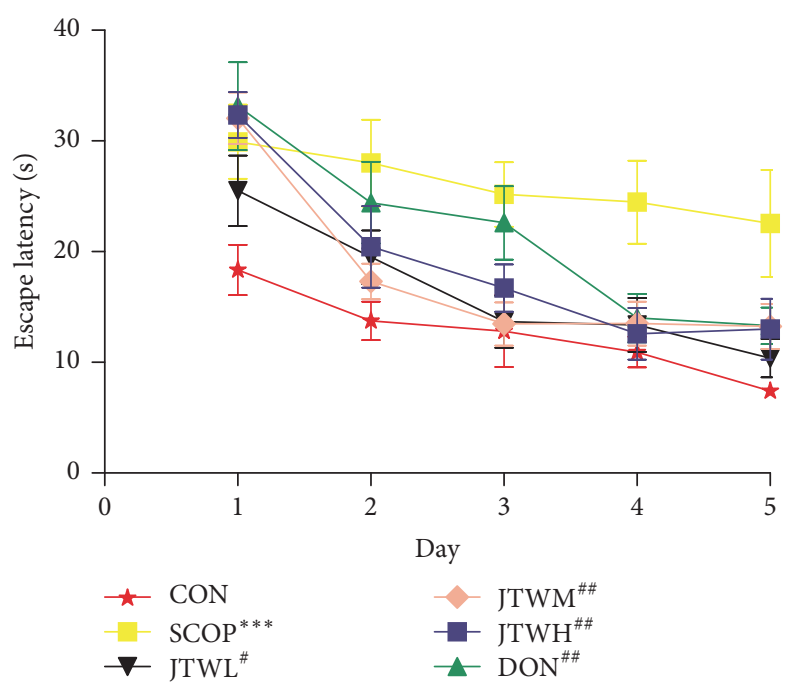

(a)

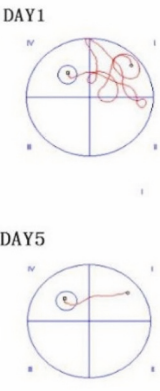

$\cos$
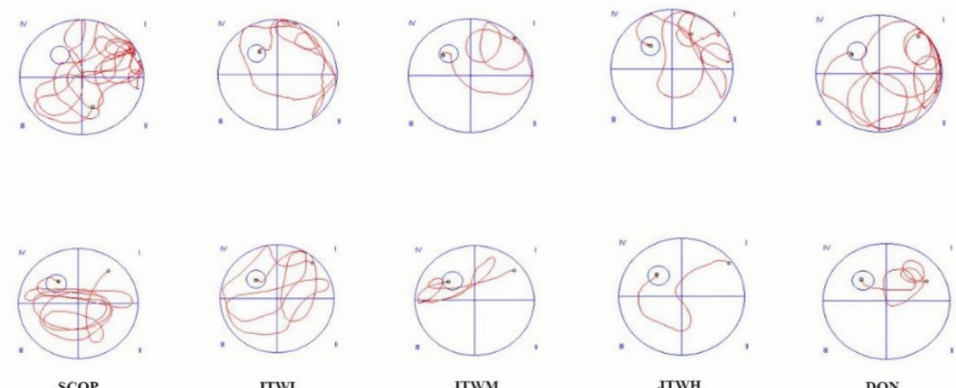

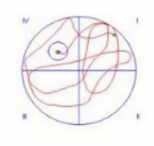

JTwL

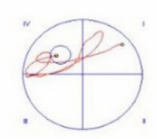

JTWM

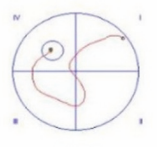

JTWH

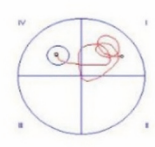

DON

(b)

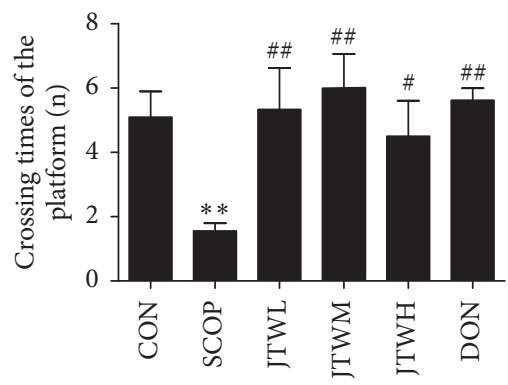

(c)

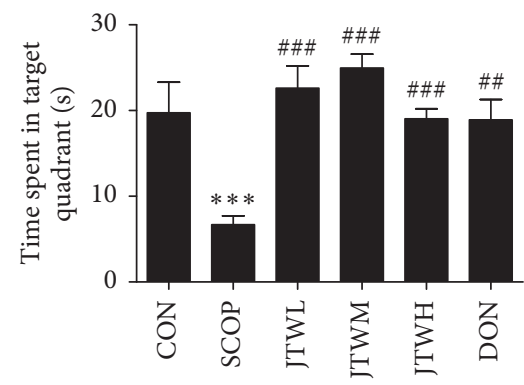

(d)

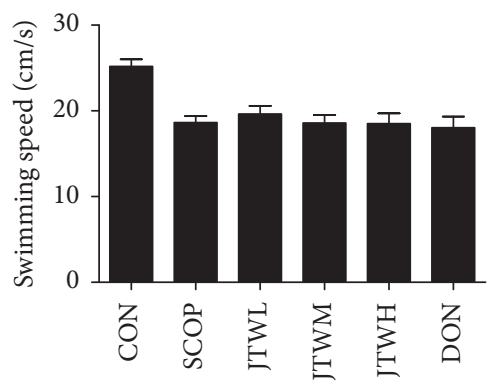

(e)

FIGURE 2: Effects of JTW prevents SCOP-treated learning and memory impairments by Morris water maze test. (a) Escape latency of five consecutive days test. (b) The swimming paths of respective groups on first and fifth day. (c) Crossing times of the target platform in the probe trial. (d) Time spent in the target quadrant in the probe trial. (e) The swimming speed in the probe trial. JTWL: Jiao-tai-wan (2.1g/kg/d); JTWM: Jiao-tai-wan (4.2g/kg/d); JTWH: Jiao-tai-wan $(8.4 \mathrm{~g} / \mathrm{kg} / \mathrm{d})$; DON: donepezil. Experimental values were expressed as mean \pm SEM $(n$ $=16$ per group). ${ }^{*} P<0.05,{ }^{* *} P<0.01,{ }^{* * *} P<0.001$ versus CON group; ${ }^{\#} P<0.05,{ }^{\# \#} P<0.01,{ }^{\# \# \#} P<0.001$ versus SCOP group.

and were deeply stained in the JTW and DON groups. To sum up, JTW could ameliorate neurodegeneration in SCOPtreated mice.

\section{Discussion}

In this study, JTW was used to evaluate the mechanism of cognitive dysfunction using Scopolamine-treated models. We found that JTW dose $(2.1 \mathrm{~g} / \mathrm{kg}, 4.2 \mathrm{~g} / \mathrm{kg}, 8.4 \mathrm{~g} / \mathrm{kg})$ slightly affect the ability of behavior, but could relieve SCOPtreated impairment of learning and memory in Kunming mice. Meanwhile, JTW could significantly ameliorate the central cholinergic nerve conduction and the injure caused by oxidative stress. In addition, JTW could alleviate neurodegeneration in the hippocampus and cortex.

The damage of cholinergic system in brain is one of the classic hypotheses of the development and progression of $\mathrm{AD}$ [27]. Scopolamine is an antagonist of $\mathrm{M}$ cholinergic 


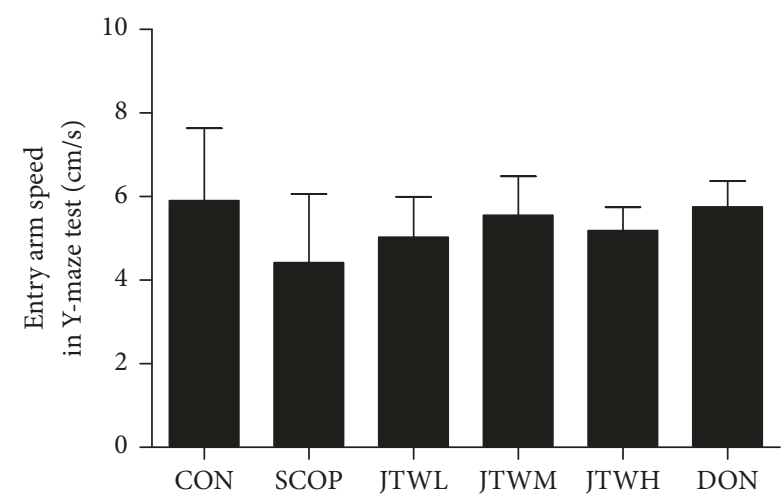

(a)

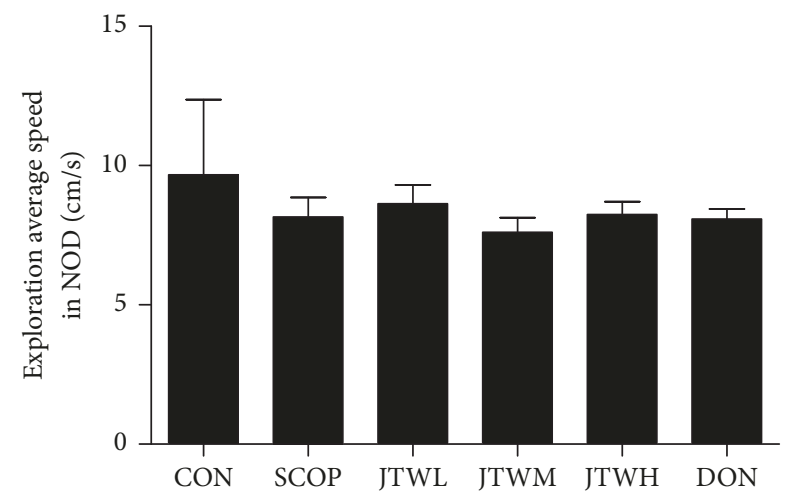

(c)

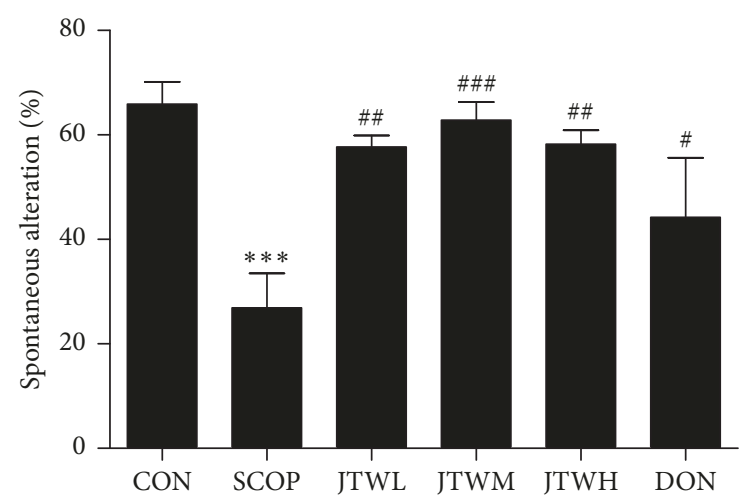

(b)

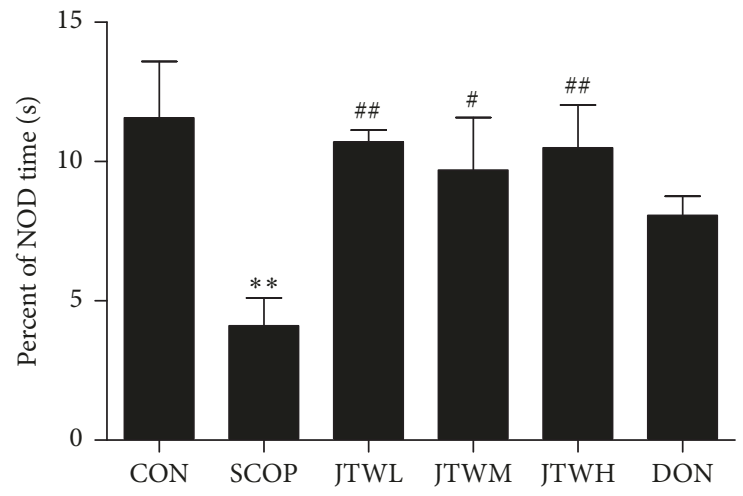

(d)

FIGURE 3: Effects of JTW prevents SCOP-treated learning and memory impairments by Y-maze and novel object discrimination. (a) Entry arm speed in Y-maze test. (b) Changes in percentage of spontaneous alternation in different groups. (c) Exploration average speed in NOD. (d) Novel object preference index. JTWL: Jiao-tai-wan (2.1g/kg/d); JTWM: Jiao-tai-wan (4.2g/kg/d); JTWH: Jiao-tai-wan (8.4 g/kg/d); DON: donepezil. Experimental values were expressed as mean $\pm \operatorname{SEM}(n=16$ per group $) .{ }^{*} P<0.05,{ }^{* *} P<0.01,{ }^{* * *} P<0.001$ versus CON group; ${ }^{\#} P<0.05,{ }^{\# \#} P<0.01,{ }^{\# \#} P<0.001$ versus SCOP group.

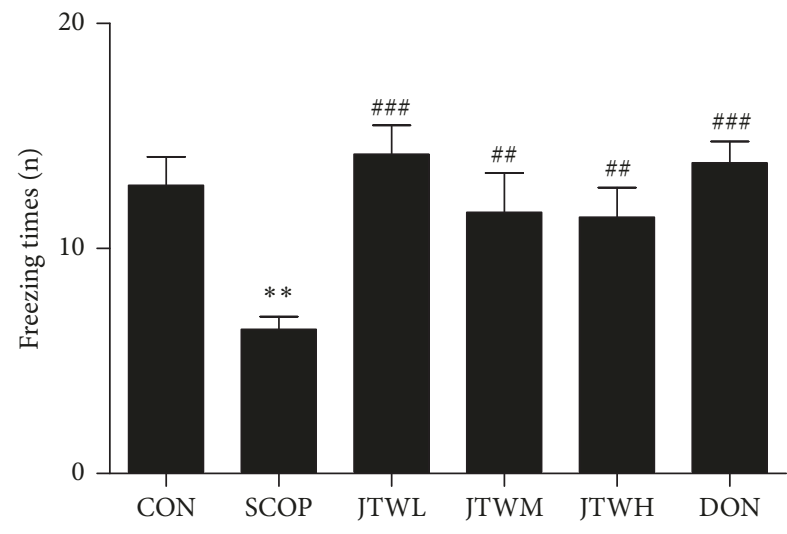

(a)

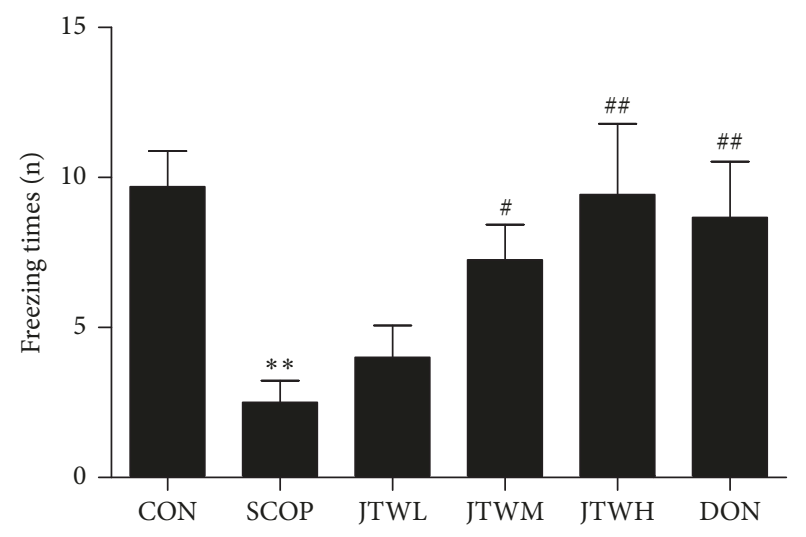

(b)

FIGURE 4: Effects of JTW prevents SCOP- treated learning and memory impairments by fearing condition test. (a) 24 hours after the first FCT is completed, the freezing times of the mouse displayed under the same environment and light stimulation but no electrical stimulation. (b) The freezing times of the mouse displayed at the first FCT under light and electrical stimulation. JTWL: Jiao-tai-wan (2.1g/kg/d); JTWM: Jiao-tai-wan (4.2g/kg/d); JTWH: Jiao-tai-wan $(8.4 \mathrm{~g} / \mathrm{kg} / \mathrm{d})$; DON: donepezil. Experimental values were expressed as mean \pm SEM $(n=16$ per group). ${ }^{*} P<0.05,{ }^{* *} P<0.01,{ }^{* * *} P<0.001$ versus CON group; ${ }^{\#} P<0.05,{ }^{\# \#} P<0.01,{ }^{\# \# \#} P<0.001$ versus SCOP group. 


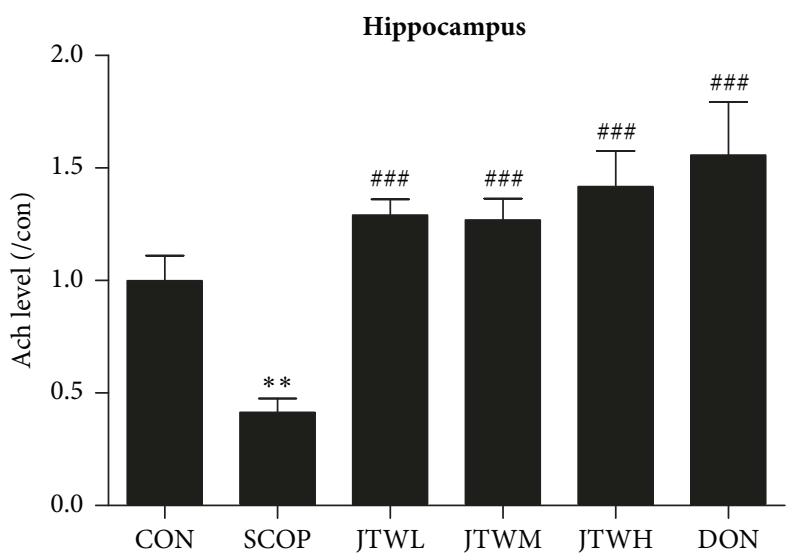

(a)

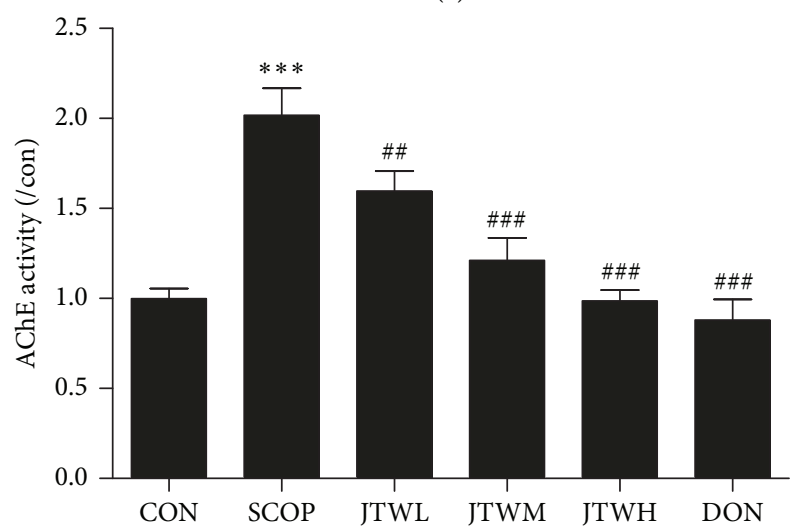

(b)

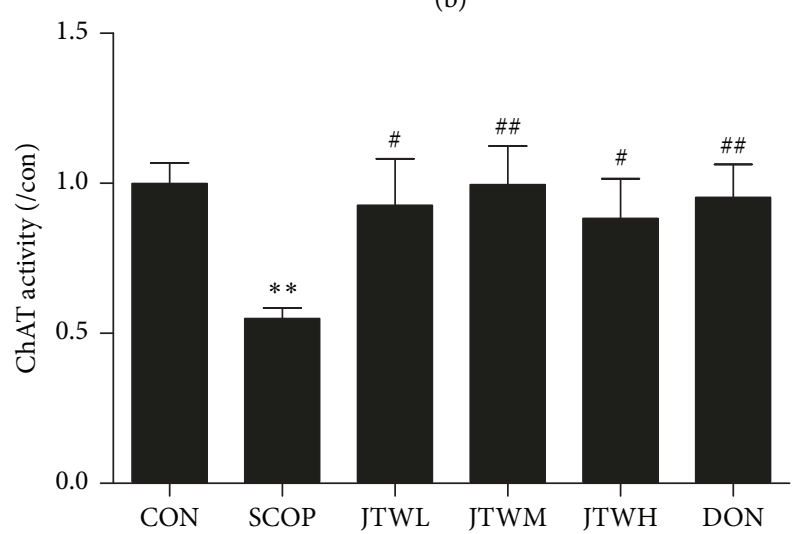

(c)

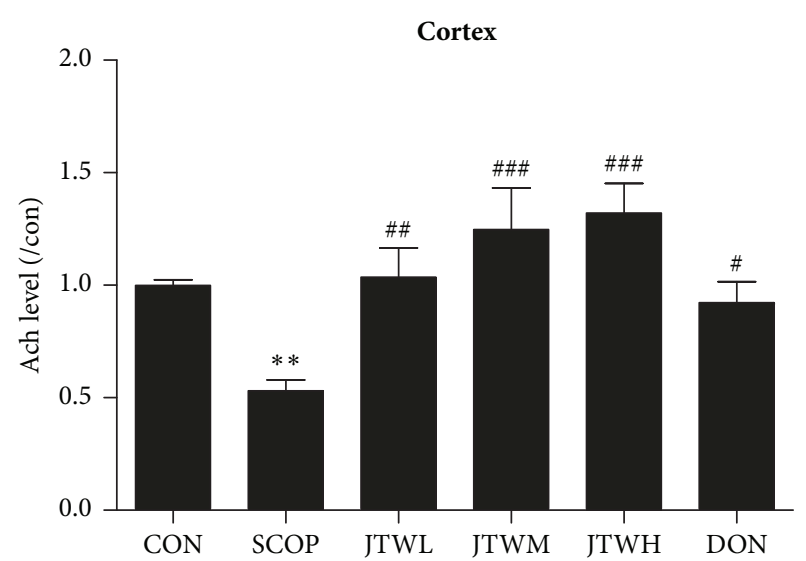

(d)

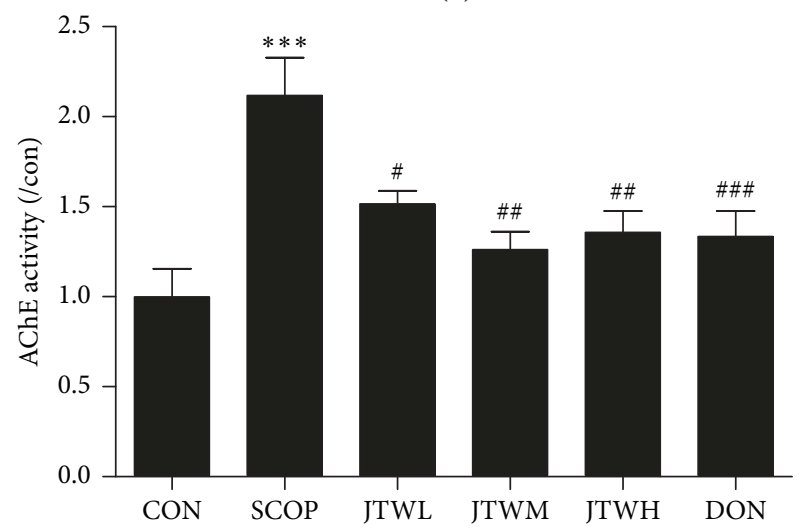

(e)

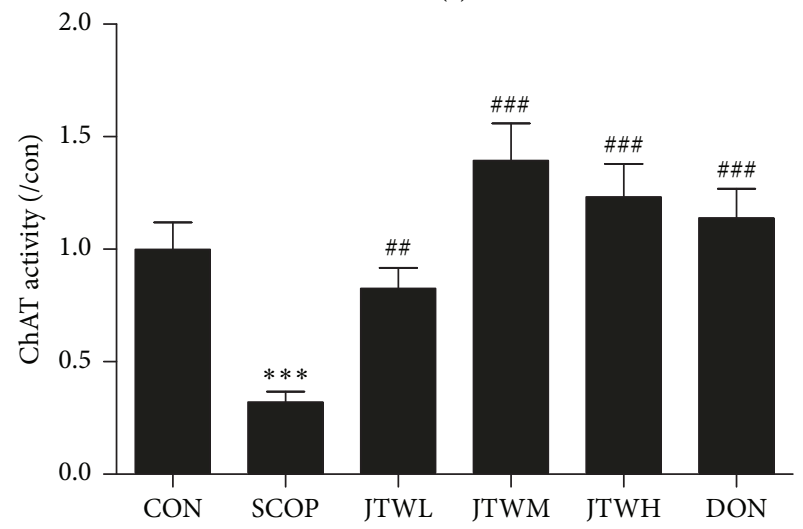

(f)

FIGURE 5: Effects of JTW improves the cholinergic nerve system in SCOP-treated Mice. The supernatant of hippocampus and cortex homogenate was used for the assay. The level of Ach and the activities of AChE and ChAT in hippocampus (a, $\mathbf{b}$ and $\mathbf{c})$. The level of Ach and the activities of AChE and ChAT in cortex (d, e and $\mathbf{f})$. JTWL: Jiao-tai-wan (2.1g/kg/d); JTWM: Jiao-tai-wan (4.2g/kg/d); JTWH: Jiao-tai-wan $(8.4 \mathrm{~g} / \mathrm{kg} / \mathrm{d})$; DON: donepezil. Experimental values were expressed as mean \pm SEM $(n=16$ per group $) .{ }^{*} P<0.05,{ }^{* *} P<0.01,{ }^{* * *} P<0.001$ versus CON group; ${ }^{\#} P<0.05,{ }^{\# \#} P<0.01,{ }^{\# \# \#} P<0.001$ versus SCOP group.

receptor. By blocking the binding of acetylcholine (ACh) with $M$ receptor, it inhibits the transmission of brain information and interferes with the formation of short-term memory, thus simulating the damaged [28] in the AD. Cholinergic system and interfering with the coding and extraction process of spatial-working memory and reference memory respectively [29-31]. Donepezil has potent acetylcholinesterase inhibition, strong selectivity and long acting time [32]. Oral administration of donepezil can inhibit the hydrolysis of acetylcholine by cholinesterase in a dose-dependent manner. Due to the high selectivity of donepezil to the acetylcholinesterase in the central nervous system, the inhibition of enzyme activity for a long time and no peripheral action, it can improve the concentration of acetylcholine in the central nervous system, especially in the cerebral cortex and basal ganglia synapses, thus improving the cognitive function. 


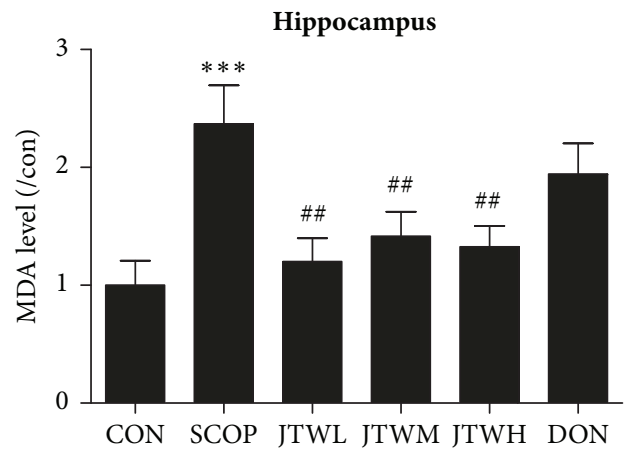

(a)

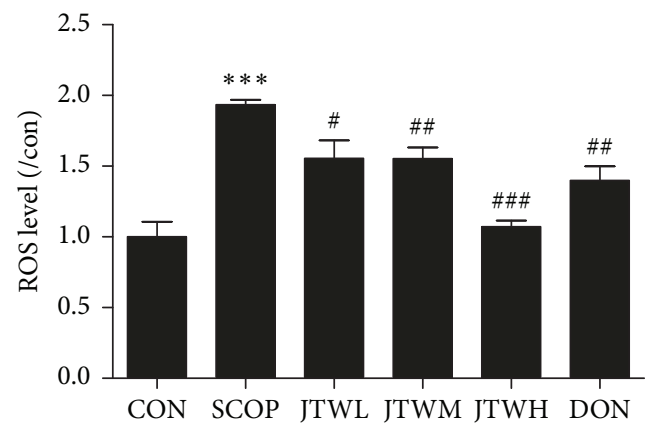

(b)

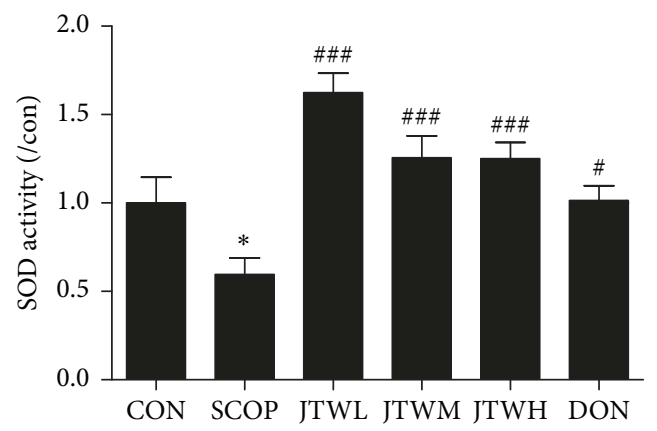

(c)

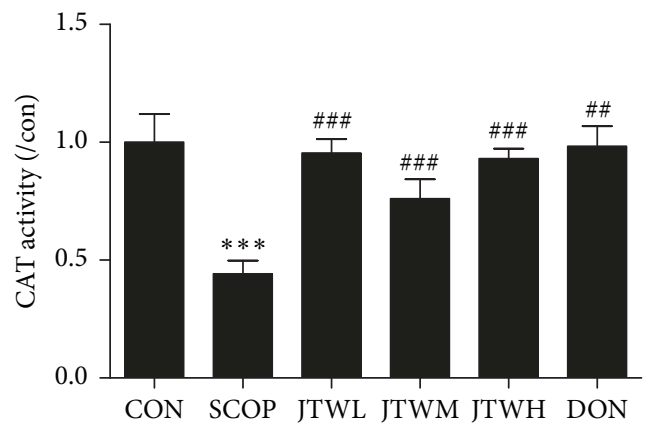

(d)

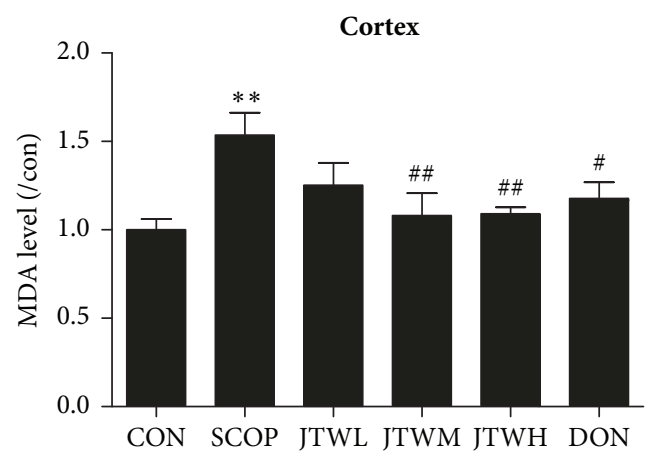

(e)

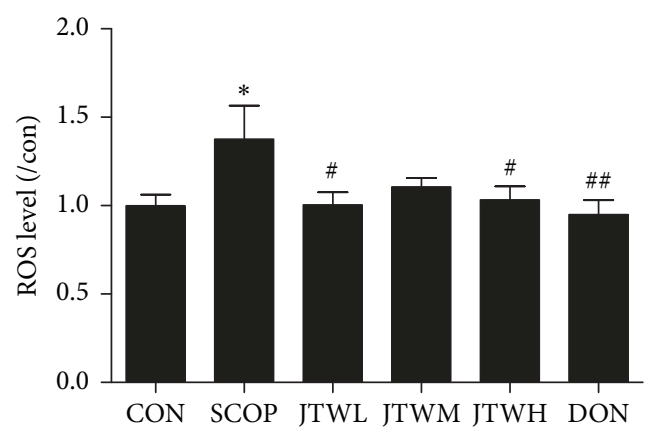

(f)

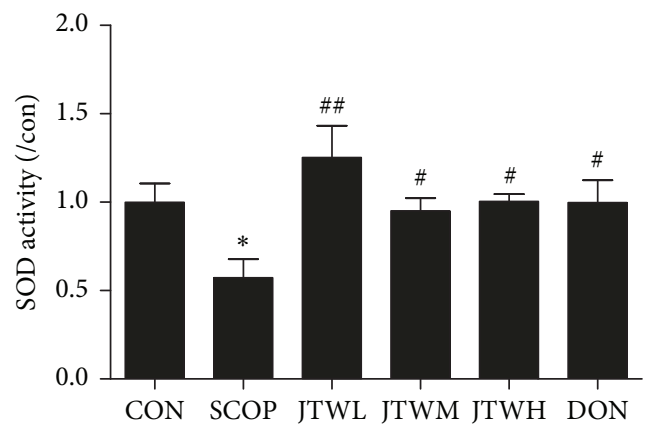

(g)

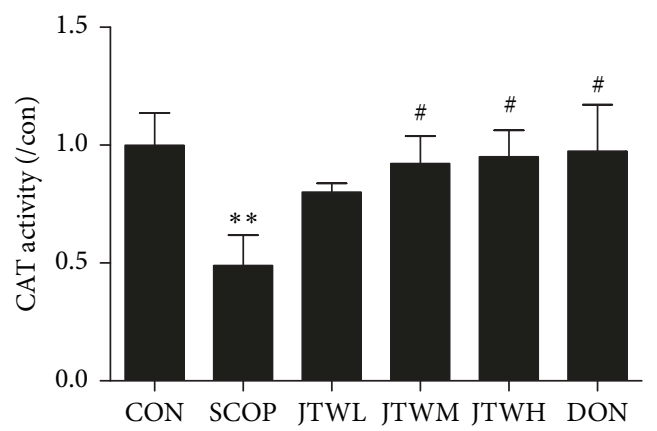

(h)

FIGURE 6: Effects of JTW improves the Oxidative Stress Status in SCOP-treated Mice. The supernatant of hippocampus and cortex homogenate was used for the assay. The level of MDA, ROS and the activities of SOD and CAT in hippocampus (a, b, c, and d). The level of MDA, ROS and the activities of SOD and CAT in cortex (e, f, g, and h). JTWL: Jiao-tai-wan (2.1g/kg/d); JTWM: Jiao-tai-wan (4.2g/kg/d); JTWH: Jiao-tai-wan $(8.4 \mathrm{~g} / \mathrm{kg} / \mathrm{d})$; DON: donepezil. Experimental values were expressed as mean \pm SEM $\left(n=16\right.$ per group). ${ }^{*} P<0.05$, ${ }^{* *} P<0.01,{ }^{* * *} P<0.001$ versus CON group; ${ }^{\#} P<0.05,{ }^{\# \#} P<0.01,{ }^{\# \# \#} P<0.001$ versus SCOP group. 

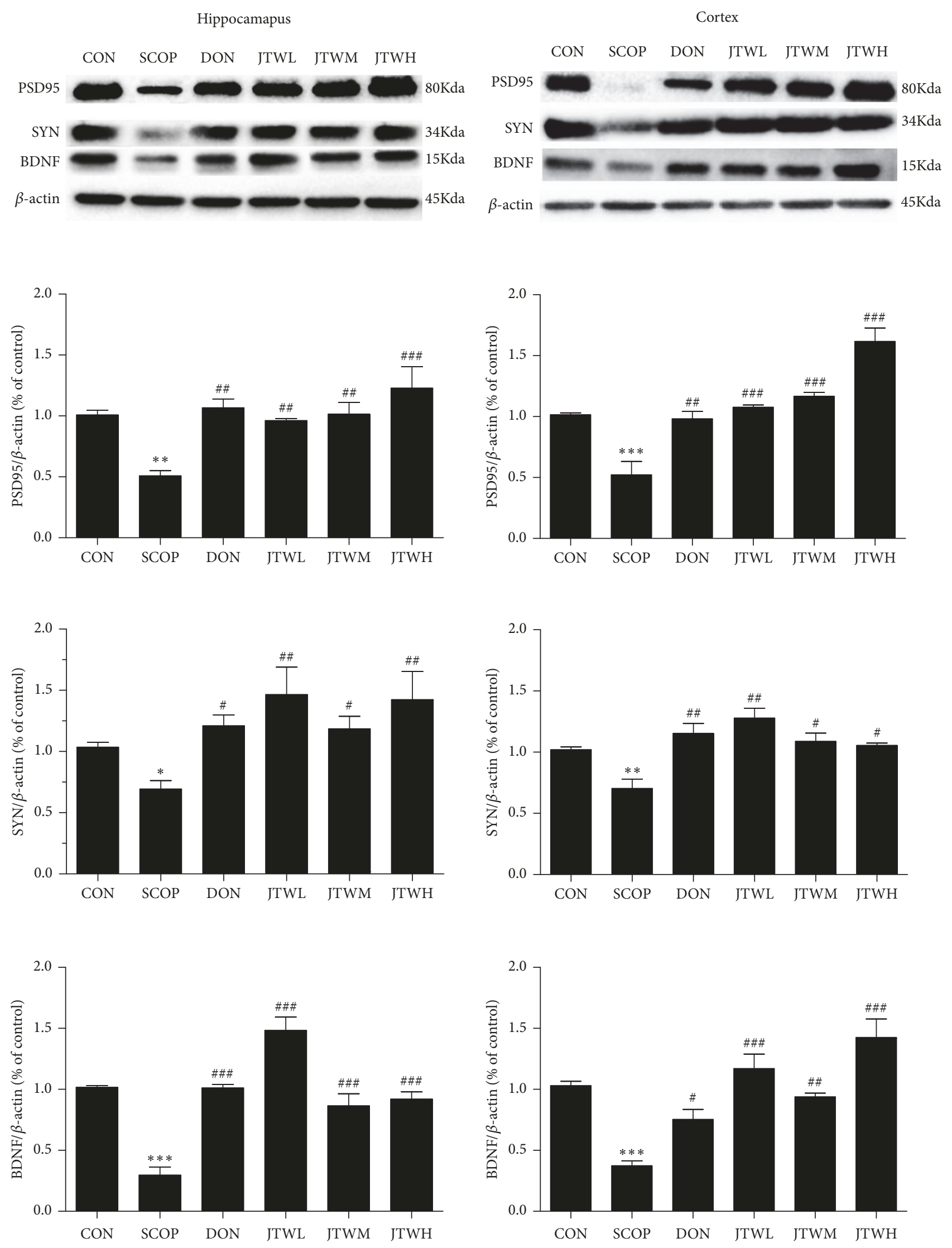

(a)

(b)

FIGURE 7: Continued. 

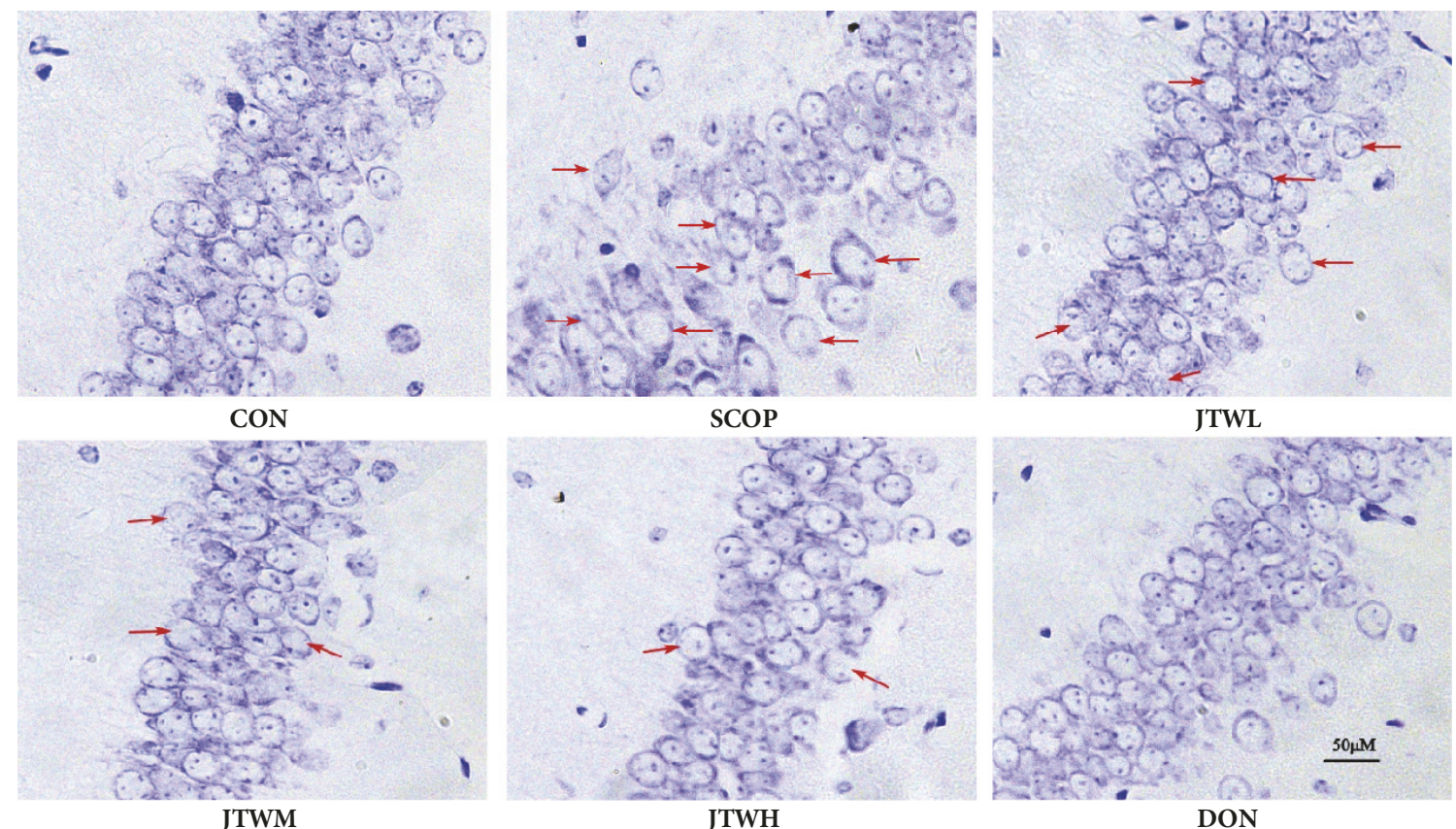

(c)

FIGURE 7: JTW improves the neurodegeneration in SCOP-treated mice. PSD95, SYN, BDNF protein levels are detected by Western Blotting in hippocampus (a) and cortex (b). (c) Nissl's staining in parietal hippocampus. Scale bar: $50 \mu \mathrm{m}$. JTWL: Jiao-tai-wan (2.1g/kg/d); JTWM: Jiao-tai-wan (4.2g/kg/d); JTWH: Jiao-tai-wan $(8.4 \mathrm{~g} / \mathrm{kg} / \mathrm{d})$; DON: donepezil. Experimental values were expressed as mean \pm SEM $(n=16$ per group). ${ }^{* * *} P<0.001$ versus CON group; ${ }^{\#} P<0.05,{ }^{\#} P<0.01,{ }^{\# \#} P<0.001$ versus $S C O P$ group.

Donepezil can prevent and control primary neuronal damage by adjusting metabolism and restoring function [33-35].

Berberine is an acetylcholinesterase inhibitor [36], whose major active constituents were extracted from traditional Chinese medicine Coptis chinensis and other plants [37], which has been used in the field of Chinese medicine and has a long-term clinical efficacy [38]. In the central nervous system diseases, Berberine has a neuroprotective function through its anti-inflammatory, anti-oxidative and anti-neuronal apoptosis pharmacological properties [3941]. After easy penetration through the blood-brain barrier, berberine is transported into neurons with a slow rate of elimination, indicating that it has a direct effect on neurons and is accumulated in the hippocampus [42]. Moreover, recent researches have indicated that berberine exerts neuroprotection in cerebral ischemia and Alzheimer's disease [43, 44]. Based on previous studies, the NF-kB signaling pathway was found as the pathogenesis of $\mathrm{AD}[45,46]$. Wenbo He [47] through the application of berberine to APP/PS1 transgenic mice, found that berberine can inhibit the activation of NF$\mathrm{kB}$ signaling pathway to delay the effect of oxidative stress and neurological inflammation, thereby effectively attenuated cognitive dysfunction. In addition, berberine can stimulate GSH synthesis and promote the activity of several antioxidative enzymes to protect cells from oxidative damage $[48,49]$. Shaktipal Patil [50] used berberine to treat ethanolinduced memory impairment in rats. The model was triggered by oxidative stress and cholinergic dysfunction. The result found that berberine can improve oxidative stress and cholinesterase activity, berberine has improved memory dysfunction caused by abnormal oxidative stress and cholinergic function. Cinnamaldehyde, a main component of traditional Chinese medicine cinnamon, is biologically active as anti-inflammatory and anti-oxidative agent [51,52], evidenced by inhibited the activity of inflammatory factors NF-kb, Nosynthase, COX-2 [53] and suppressed release of noradernaline in guinea-pig ileum myenteric nerve terminals $[54,55]$. In the experiment, Yang D [19] found cinnamaldehyde have anti-oxidation and anti-inflammatory effects, and it had repair and protection effects on the injured neurons of rat dorsal ganglion. To sum up, the main components of JTW can improve oxidative stress, neuroinflammation, cholinergic abnormalities and other factors related to cognitive function.

Cognitive dysfunction as a moderate state between normal cognition and dementia, which has become a major public health issue due to its high socioeconomic status [56] which is characterized as the gradual loss of learning ability and cognitive function, as well as memory impairment, which are considered as typical symptoms of $\mathrm{AD}[57,58]$. A vast number of experimental studies have suggested that cholinergic neurotransmission dysfunction in the cerebral hippocampus and cortex plays an important role in cognitive impairment [4]. The central nervous system of $\mathrm{AD}$ patients shows abnormalities in cholinergic function are closely related to cognitive dysfunction [59]. Some studies [7, 60] have found that when treating cognitive dysfunction, the use of drugs that activate the cholinergic system can promote learning and memory functions in animals. Scopolamine 
(SCOP) as inducing amnesia in mammals, which is a nonselective anti-muscarinic that penetrates the blood-brain barrier (BBB) and produces a model similar to early $\mathrm{AD}$ symptoms $[61,62]$. SCOP inhibits central cholinergic neurons by blocking two major systems of the forebrain cholinergic activity (nuclei basalis-cerebral cortex and septohippocampal pathways), resulting in accurate, repeatable and transient memory damage to normal humans and healthy animals [63, 64]. We introduced the following behavior tests to observe the SCOP-induced cognitive dysfunction model: Morris water maze test, Y-maze, NOD and FCT to measure the memory deficits of the SCOP-treated mice. Results show that the SCOP group which accepted JTW treatment have obvious ameliorated effect on learning ability and cognitive function, as well as memory impairment. The SCOP group have a longer latency, a few of crossing times of the platform and time spent in target quadrant, cognitive function have not been improved.

Some studies have also reported that the treatment of cholinergic neurotransmitters is the most promising treatment for cognitive dysfunction caused by SCOP [65]. Acetylcholine (ACh) [66] is considered to be the most important neurotransmitter involved in the regulation of cognitive functions. After releasing from the presynaptic neuron, Ach, the neurotransmitter, was gathered into the synaptic cleft, followed by binding to the Ach receptors on the postsynaptic membrane, and the signal from the nerve was relayed during the process [67]. The termination of the signal transmission was conducted by hydrolyzation of ACh located on the postsynaptic membrane [68]. AChE, one of the most important enzyme in the family serine hydrolase involved in the hydrolytic cleavage of Ach, depletes the levels of ACh involved in memory and learning [69]. Recent studies have found that AChE increased its activity is a major cause of neurodegenerative diseases. Through electron emission tomography, AChE was severely increased in the forebrain of AD patients [70,71]. ChAT is a key part of the synthesis of cholinergic neurons, which participates in Ach synthesis. The protein express and activity of ChAT decreased at early stages of $\mathrm{AD}$ and cognitive impairment $[72,73]$. The decline in the activity of choline acetyltransferase (ChAT) and acetylcholine esterase (AChE), as well as the release amount of acetylcholine (ACh) often appear in the SCOPtreated mice [74]. After inspection of the cholinergic system in SCOP-treated mice in the study, we concluded that the level of ACh and the activity of ChAT were increased while the activity of AChE was decreased in the JTW groups compared with the SCOP group.

Brain tissue is most vulnerable to the harmful effects of reactive oxygen species. Reactive oxygen species (ROS) may lead to the process of lipid peroxidation in neuron degeneration, which is most prominent in the central cholinergic pathway. Because the ROS has a high oxygen consumption rate and can reduce the capacity of the antioxidant defense system $[75,76]$. Some research proposed that ROS generated by oxidative stress is one of the pathogeneses of dementia, including $\mathrm{AD}$ and $\mathrm{CD}$. Yuan $\mathrm{Hu}$ [77] have found that when the supply of oxygen to the brain was low, ROS production, which promote membrane lipid oxidation were extremely increased, DNA, and essential proteins, leading to neuronal apoptosis and cognitive dysfunction. Malondialdehyde (MDA) was used as an end product of lipid peroxidation and commonly be used to demonstrate the occurrence of increased peroxidative stress [78], which was mainly distributed in various parts of the brain, such as the cortex, the hippocampus, the striatum, and the whole brain [79]. At the same time, the degree of oxidative stress determines the severity of this cognitive impairment. Superoxide dismutase (SOD) is an endogenous antioxidant enzyme, when the superoxide-oxidase level was exhausted, the resulting superoxide can directly cause cell damage [80]. Catalase as a parameter of oxidative stress in brain tissue and was used to study homogenates of cognitive dysfunction in animals [81]. Studies reported that when different parts of the brain were exposed to various chemicals, it causes a significant drop in CAT and causing cognitive impairment [80]. The activities of the antioxidant enzymes CAT and SOD were significantly diminished due to SCOP- treated cognitive impairment [82]. In the study, robustly increased ROS and MDA levels and stifled activities of SOD and CAT were detected in the SCOPtreated group. Furthermore, the ROS and MDA levels were inhibited while the SOD and CAT activities were increased in both hippocampus and cortex in the JTW-treated groups.

Synapses, phosphoproteins, was associated with synaptic vesicles participated in neuronal development and neurotransmitter release [83, 84]. Many research have reported that impaired cognitive ability, learning ability and memory are alternately associated with synapses [85]. Postsynaptic density protein 95 (PSD95), mainly in the hippocampus and prefrontal cortex, are closely related to cognitive disorders [86]. Synaptophysin (SYN) is a protein that has an important influence on cognitive function and is located in the presynaptic vesicle [86]. Brain-derived neurotrophic factor (BDNF) is one of the most important brain - derived neurotrophic factors in the brain. It is mainly involved in the response to learning, exercise, memory, and external stress [87]. BDNF is produced during the process of synaptic pruning and apoptosis, and regulates neurotransmission through the involvement of synapses, depending on the presence of synapses [88-90]. Increased expression of BDNF can reduce neuronal synaptic damage in nervous system diseases [91]. In this study, there were certain neuronal damages. In the JTW and DON groups, the low expressions of PSD95, SYN and BDNF were reinstated. In addition, Nissl's staining was applied to prove the neuroprotective effect of JTW. The above results demonstrated that the protective effect of JTW was able to inhibit SCOP- treated neurodegeneration in the hippocampus and cortex.

\section{Conclusion}

We validated that the treatment of JTW can effectively promote the ability of spatial recognition, learning and memory, and the memory ability of fresh things of SCOP-treated mice. Then, the activity of acetylcholinesterase (AChE) was effectively decreased; the activity of choline acetyltransferase (ChAT) and concentration of acetylcholine (Ach) were improved after JTW treatment in SCOP-treated mice. 
In addition, JTW effectively ameliorated oxidative stress because of decreased the levels of malondialdehyde (MDA) and reactive oxygen species (ROS) and increased the activities of superoxide dismutase (SOD) and catalase (CAT) in hippocampus and cortex. At last, JTW promote the expressions of neurotrophic factors including postsynaptic density protein 95 (PSD95) and synaptophysin (SYN) and brainderived neurotrophic factor (BDNF). As part of multi-target strategies, JTW might be a potential anti-AD drug. However, further mechanisms and clinical trials are warranted for a better understanding and clinical practice of JTW.

\section{Data Availability}

All original data in the manuscript are available from the author.

\section{Conflicts of Interest}

The authors declare that they have no conflicts of interest.

\section{Authors' Contributions}

Xin-Chen Wang and Yu-Min Xu contributed equally to this work.

\section{Acknowledgments}

This work was supported by the Guangdong Provincial Major Science and Technology for Special Program of China (no. 2012A080202017; no. 2015A030302072), South China Chinese Medicine Collaborative Innovation Center and Guangzhou Science Technology and Innovation Commission Technology Research Projects.

\section{References}

[1] H. Okada, Y. Ouchi, M. Ogawa et al., "Alterations in $\alpha 4 \beta 2$ nicotinic receptors in cognitive decline in Alzheimer's aetiopathology," Brain, vol. 136, no. 10, pp. 3004-3017, 2013.

[2] R. Goekoop, P. Scheltens, F. Barkhof, and S. A. R. B. Rombouts, "Cholinergic challenge in Alzheimer patients and mild cognitive impairment differentially affects hippocampal activation A pharmacological fMRI study," Brain, vol. 129, no. 1, pp. 141$157,2006$.

[3] B. H. Chen, J. H. Park, T. Lee et al., "Melatonin attenuates scopolamine-induced cognitive impairment via protecting against demyelination through BDNF-TrkB signaling in the mouse dentate gyrus," Chemico-Biological Interactions, vol. 285, pp. 8-13, 2018.

[4] R. Schliebs and T. Arendt, "The significance of the cholinergic system in the brain during aging and in Alzheimer's disease," Journal of Neural Transmission, vol. 113, no. 11, pp. 1625-1644, 2006.

[5] J. C. Morris, "Challenging assumptions about Alzheimer's disease: Mild cognitive impairment and the cholinergic hypothesis," Annals of Neurology, vol. 51, no. 2, pp. 143-144, 2002.

[6] P. J. Ghumatkar, S. P. Patil, P. D. Jain, R. M. Tambe, and S. Sathaye, "Nootropic, neuroprotective and neurotrophic effects of phloretin in scopolamine induced amnesia in mice," Pharmacology Biochemistry \& Behavior, vol. 135, pp. 182-191, 2015.

[7] I. H. Jung, H. E. Lee, S. J. Park et al., "Ameliorating effect of spinosin, a C-glycoside flavonoid, on scopolamine-induced memory impairment in mice," Pharmacology Biochemistry \& Behavior, vol. 120, pp. 88-94, 2014.

[8] C. Haense, E. Kalbe, K. Herholz et al., "Cholinergic system function and cognition in mild cognitive impairment," Neurobiology of Aging, vol. 33, no. 5, pp. 867-877, 2012.

[9] N. Fujiwara, J. Shimizu, K. Takai et al., "Restoration of spatial memory dysfunction of human APP transgenic mice by transplantation of neuronal precursors derived from human iPS cells," Neuroscience Letters, vol. 557, pp. 129-134, 2013.

[10] K. Yanagiha, K. Ishii, and A. Tamaoka, "Acetylcholinesterase inhibitor treatment alleviated cognitive impairment caused by delayed encephalopathy due to carbon monoxide poisoning Two case reports and a review of the literature," Medicine (United States), vol. 96, no. 8, Article ID e6125, 2017.

[11] D. Park, H. J. Lee, S. S. Joo et al., "Human neural stem cells over-expressing choline acetyltransferase restore cognition in rat model of cognitive dysfunction," Experimental Neurology, vol. 234, no. 2, pp. 521-526, 2012.

[12] J. Chen, X. Zhang, Y. Wang, Y. Ye, and Z. Huang, "Formononetin promotes proliferation that involves a feedback loop of microRNA-375 and estrogen receptor alpha in estrogen receptor-positive cells," Molecular Carcinogenesis, vol. 55, no. 3, pp. 312-319, 2016.

[13] M.-M. Chen, G.-W. Zhao, P. He et al., "Improvement in the neural stem cell proliferation in rats treated with modified "shengyu" decoction may contribute to the neurorestoration," Journal of Ethnopharmacology, vol. 165, pp. 9-19, 2015.

[14] H.-F. Ji and L. Shen, "Berberine: A potential multipotent natural product to combat Alzheimer's disease," Molecules, vol. 16, no. 8, pp. 6732-6740, 2011.

[15] T. Sartorius, A. Peter, N. Schulz et al., "Cinnamon extract improves insulin sensitivity in the brain and lowers liver fat in mouse models of obesity," PLoS ONE, vol. 9, no. 3, Article ID e92358, 2014

[16] P. Wang, W. Liao, J. Fang et al., "A glucan isolated from flowers of Lonicera japonica Thunb. Inhibits aggregation and neurotoxicity of $\mathrm{A} \beta_{42}$," Carbohydrate Polymers, vol. 110, pp. 142147,2014

[17] J.-J. Kim, J. Jiang, and D.-W. Shim, "Anti-inflammatory and antiallergic effects of Agrimonia pilosa Ledeb extract on murine cell lines and OVA-induced airway inflammation," Journal of Ethnopharmacology, vol. 140, no. 2, pp. 213-221, 2012.

[18] F. Zhu, Y. Ma, and Y. Sun, "Effect of berberine on the expression of insulin degrading enzyme in an Alzheimers disease rat model," Chinese Journal of Neuromedicine, vol. 9, no. 12, pp. 1201-1203, 2012.

[19] D. Yang, X.-C. Liang, Y. Shi et al., "Anti-oxidative and antiinflammatory effects of cinnamaldehyde on protecting high glucose-induced damage in cultured dorsal root ganglion neurons of rats," Chinese Journal of Integrative Medicine, vol. 22, no. 1, pp. 19-27, 2016.

[20] C. V. Vorhees and M. T. Williams, "Morris water maze: procedures for assessing spatial and related forms of learning and memory," Nature Protocols, vol. 1, no. 2, pp. 848-858, 2006.

[21] J. Bouayed, F. Desor, H. Rammal et al., "Effects of lactational exposure to benzo $[\alpha]$ pyrene $(\mathrm{B}[\alpha] \mathrm{P})$ on postnatal neurodevelopment, neuronal receptor gene expression and behaviour in mice," Toxicology, vol. 259, no. 3, pp. 97-106, 2009. 
[22] A. S. R. Sierksma, D. L. A. Van Den Hove, F. Pfau et al., "Improvement of spatial memory function in APPswe/PS1dE9 mice after chronic inhibition of phosphodiesterase type 4D," Neuropharmacology, vol. 77, pp. 120-130, 2014.

[23] P. Tucci, E. Mhillaj, M. G. Morgese et al., "Memantine prevents memory consolidation failure induced by soluble beta amyloid in rats," Frontiers in Behavioral Neuroscience, vol. 8, p. 332, 2014.

[24] M. Antunes and G. Biala, "The novel object recognition memory: neurobiology, test procedure, and its modifications," Cognitive Processing, vol. 13, no. 2, pp. 93-110, 2012.

[25] C. Lemini, M. Estela Avila, M. Medina et al., "Proliferative Properties of $17 \beta$-aminoestrogens in MCF-7 Human Breast Cancer Cells," Basic \& Clinical Pharmacology \& Toxicology, vol. 120, no. 3, pp. 235-242, 2017.

[26] G. D. Gale, R. D. Yazdi, A. H. Khan, A. J. Lusis, R. C. Davis, and D. J. Smith, "A genome-wide panel of congenic mice reveals widespread epistasis of behavior quantitative trait loci," Molecular Psychiatry, vol. 14, no. 6, pp. 631-645, 2009.

[27] W. Chen, X. Cheng, J. Chen et al., "Lycium barbarum polysaccharides prevent memory and neurogenesis impairments in scopolamine-treated rats," PLoS ONE, vol. 9, no. 2, Article ID e88076, 2014.

[28] S. Hescham, Y. Temel, J. Casaca-Carreira et al., "A neuroanatomical analysis of the effects of a memory impairing dose of scopolamine in the rat brain using cytochrome $c$ oxidase as principle marker," Journal of Chemical Neuroanatomy, vol. 5960, pp. 1-7, 2014.

[29] K. Vales and A. Stuchlik, "Central muscarinic blockade interferes with retrieval and reacquisition of active allothetic place avoidance despite spatial pretraining," Behavioural Brain Research, vol. 161, no. 2, pp. 238-244, 2005.

[30] E. Von Linstow Roloff, D. Harbaran, J. Micheau, B. Platt, and G. Riedel, "Dissociation of cholinergic function in spatial and procedural learning in rats," Neuroscience, vol. 146, no. 3, pp. 875-889, 2007.

[31] D. Saar, Y. Grossman, and E. Barkai, "Long-lasting cholinergic modulation underlies rule learning in rats," The Journal of Neuroscience, vol. 21, no. 4, pp. 1385-1392, 2001.

[32] H. Ogura, T. Kosasa, S. Araki, and Y. Yamanishi, "Pharmacological properties of donepezil hydrochloride (Aricept ${ }^{\circledR}$ ), a drug for Alzheimer's disease," Folia Pharmacologica Japonica, vol. 115, no. 1, pp. 45-51, 2000.

[33] R. T. Staff, H. G. Gemmell, M. F. Shanks, A. D. Murray, and A. Venneri, "Changes in the rcbf images of patients with alzheimer's disease receiving donepezil therapy," Nuclear Medicine Communications, vol. 21, no. 1, pp. 37-41, 2000.

[34] I. Hatip-Al-Khatib, A. Takashi, N. Egashira, K. Iwasaki, and M. Fujiwara, "Comparison of the effect of TAK-147 (zanapezil) and E-2020 (donepezil) on extracellular acetylcholine level and blood flow in the ventral hippocampus of freely moving rats," Brain Research, vol. 1012, no. 1-2, pp. 169-176, 2004.

[35] J. S. Meyer, M. H. Chowdhury, G. Xu, Y.-S. Li, and M. Quach, "Donepezil treatment of vascular dementia," Annals of the New York Academy of Sciences, vol. 977, pp. 482-486, 2002.

[36] K. Ingkaninan, P. Phengpa, S. Yuenyongsawad, and N. Khorana, "Acetylcholinesterase inhibitors from Stephania venosa tuber," Journal of Pharmacy and Pharmacology, vol. 58, no. 5, pp. 695700, 2006.

[37] M. Imanshahidi and H. Hosseinzadeh, "Pharmacological and therapeutic effects of Berberis vulgaris and its active constituent, berberine," Phytotherapy Research, vol. 22, no. 8, pp. 999-1012, 2008.
[38] M. Huang, S. Chen, Y. Liang, and Y. Guo, “The Role of Berberine in the Multi-Target Treatment of Senile Dementia," Curr Top Med Chem, vol. 16, no. 8, pp. 867-873, 2016.

[39] J.-S. Hong, Y.-K. Chu, H. Lee et al., "Effects of berberine on hippocampal neuronal damage and matrix metalloproteinase-9 activity following transient global cerebral ischemia," Journal of Neuroscience Research, vol. 90, no. 2, pp. 489-497, 2012.

[40] X. Zhang, X. Zhang, C. Wang et al., "Neuroprotection of early and short-time applying berberine in the acute phase of cerebral ischemia: Up-regulated pAkt, pGSK and pCREB, downregulated NF- $\kappa \mathrm{B}$ expression, ameliorated BBB permeability," Brain Research, vol. 1459, pp. 61-70, 2012.

[41] L. Gu, N. Li, and W. Yu, "Berberine reduces rat intestinal tight junction injury induced by ischemia-reperfusion associated with the suppression of inducible nitric oxide synthesis," American Journal of Chinese Medicine, vol. 41, no. 6, pp. 12971312, 2013.

[42] X. Wang, R. Wang, D. Xing et al., "Kinetic difference of berberine between hippocampus and plasma in rat after intravenous administration of Coptidis rhizoma extract," Life Sciences, vol. 77, no. 24, pp. 3058-3067, 2005.

[43] E. N. Simões Pires, R. L. Frozza, J. B. Hoppe, B. D. M. Menezes, and C. G. Salbego, "Berberine was neuroprotective against an in vitro model of brain ischemia: Survival and apoptosis pathways involved," Brain Research, vol. 1557, pp. 26-33, 2014.

[44] N. Panahi, M. Mahmoudian, P. Mortazavi, and G. S. Hashjin, "Effects of berberine on $\beta$-secretase activity in a rabbit model of Alzheimer's disease," Archives of Medical Science, vol. 9, no. 1, pp. 146-150, 2013.

[45] Z. Cai, C. Wang, and W. Yang, "Role of berberine in Alzheimer's disease," Neuropsychiatric Disease and Treatment, vol. 12, pp. 2509-2520, 2016.

[46] U. Kaur, P. Banerjee, A. Bir, M. Sinha, A. Biswas, and S. Chakrabarti, "Reactive oxygen species, redox signaling and neuroinflammation in Alzheimer's disease: The NF- $\kappa$ B connection," Current Topics in Medicinal Chemistry, vol. 15, no. 5, pp. 446-457, 2015.

[47] W. He, C. Wang, Y. Chen, Y. He, and Z. Cai, "Berberine attenuates cognitive impairment and ameliorates tau hyperphosphorylation by limiting the self-perpetuating pathogenic cycle between NF- $\kappa \mathrm{B}$ signaling, oxidative stress and neuroinflammation," Pharmacological Reports, vol. 69, no. 6, pp. 13411348, 2017.

[48] L. Tang, W. Wei, L. Chen, and S. Liu, "Effects of berberine on diabetes induced by alloxan and a high-fat/high-cholesterol diet in rats," Journal of Ethnopharmacology, vol. 108, no. 1, pp. 109115, 2006.

[49] H. Kalalian-Moghaddam, T. Baluchnejadmojarad, M. Roghani, F. Goshadrou, and A. Ronaghi, "Hippocampal synaptic plasticity restoration and anti-apoptotic effect underlie berberine improvement of learning and memory in streptozotocindiabetic rats," European Journal of Pharmacology, vol. 698, no. 1-3, pp. 259-266, 2013.

[50] S. Patil, S. Tawari, D. Mundhada, and S. Nadeem, "Protective effect of berberine, an isoquinoline alkaloid ameliorates ethanol-induced oxidative stress and memory dysfunction in rats," Pharmacology Biochemistry \& Behavior, vol. 136, Article ID 72225, pp. 13-20, 2015.

[51] P. Subash-Babu, A. A. Alshatwi, and S. Ignacimuthu, "Beneficial antioxidative and antiperoxidative effect of cinnamaldehyde protect streptozotocin-induced pancreatic $\beta$-cells damage in 
wistar rats," Biomolecules \& Therapeutics, vol. 22, no. 1, pp. 4754, 2014.

[52] L. K. Chao, K.-F. Hua, H.-Y. Hsu et al., "Cinnamaldehyde inhibits pro-inflammatory cytokines secretion from monocytes/macrophages through suppression of intracellular signaling," Food and Chemical Toxicology, vol. 46, no. 1, pp. 220-231, 2008.

[53] J.-T. Cheng, I.-M. Liu, W.-C. Huang, and D. H. Kou, "Stimulatory effect of trans-cinnamaldehyde on noradrenaline secretion in guinea-pig ileum myenteric nerve terminals," Life Sciences, vol. 66, no. 11, pp. 981-990, 2000.

[54] D. H. Kim, C. H. Kim, M.-S. Kim et al., "Suppression of agerelated inflammatory NF-kappaB activation by cinnamaldehyde," Biogerontology, vol. 8, no. 5, pp. 545-554, 2007.

[55] H. S. Youn, J. K. Lee, Y. J. Choi et al., "Cinnamaldehyde suppresses toll-like receptor 4 activation mediated through the inhibition of receptor oligomerization," Biochemical Pharmacology, vol. 75, no. 2, pp. 494-502, 2008.

[56] J. Wattanathorn, S. Muchimapura, W. Thukham-Mee, K. Ingkaninan, and S. Wittaya-Areekul, "Mangifera indica fruit extract improves memory impairment, cholinergic dysfunction, and oxidative stress damage in animal model of mild cognitive impairment," Oxidative Medicine and Cellular Longevity, vol. 2014, Article ID 132097, 7 pages, 2014.

[57] R. Brookmeyer, E. Johnson, K. Ziegler-Graham, and H. M. Arrighi, "Forecasting the global burden of Alzheimer's disease," Alzheimer's \& Dementia, vol. 3, no. 3, pp. 186-191, 2007.

[58] M. S. Parihar and T. Hemnani, "Alzheimer's disease pathogenesis and therapeutic interventions," Journal of Clinical Neuroscience, vol. 11, no. 5, pp. 456-467, 2004.

[59] E. J. Mufson, S. E. Counts, S. E. Perez, and S. D. Ginsberg, "Cholinergic system during the progression of Alzheimer's disease: Therapeutic implications," Expert Review of Neurotherapeutics, vol. 8, no. 11, pp. 1703-1718, 2008.

[60] F. Guida, L. Luongo, F. Marmo et al., "Palmitoylethanolamide reduces pain-related behaviors and restores glutamatergic synapses homeostasis in the medial prefrontal cortex of neuropathic mice," Molecular Brain, vol. 8, no. 1, article no. 47, 2015.

[61] S. Bihaqi, A. Singh, and M. Tiwari, "Supplementation of Convolvulus pluricaulis attenuates scopolamine-induced increased tau and Amyloid precursor protein $(\mathrm{A} \beta \mathrm{PP})$ expression in rat brain," Indian Journal of Pharmacology, vol. 44, no. 5, pp. 593598, 2012.

[62] B. Budzyńska, A. Boguszewska-Czubara, M. Kruk-Slomka et al., "Effects of imperatorin on scopolamine-induced cognitive impairment and oxidative stress in mice," Psychopharmacology, vol. 232, no. 5, pp. 931-942, 2015.

[63] B. Lee, J. Park, S. Kwon et al., "Effect of wild ginseng on scopolamine-induced acetylcholine depletion in the rat hippocampus," Journal of Pharmacy and Pharmacology, vol. 62, no. 2, pp. 263-271, 2010.

[64] M. Prior, R. Dargusch, J. L. Ehren, C. Chiruta, and D. Schubert, "The neurotrophic compound J147 reverses cognitive impairment in aged Alzheimer's disease mice," Alzheimer's Research and Therapy, vol. 5, no. 3, p. 25, 2013.

[65] H. Soreq and S. Seidman, "Acetylcholinesterase: new roles for an old actor," Nature Reviews Neuroscience, vol. 2, no. 4, pp. 294302, 2001.

[66] P. E. Gold, "Acetylcholine: Cognitive and brain functions," Neurobiology of Learning and Memory, vol. 80, no. 3, p. 177, 2003.
[67] R. Schliebs and T. Arendt, "The cholinergic system in aging and neuronal degeneration," Behavioural Brain Research, vol. 221, no. 2, pp. 555-563, 2011.

[68] T. Liu, Z. Xia, W.-W. Zhang et al., "Bis(9)-(-)-nor-meptazinol as a novel dual-binding AChEI potently ameliorates scopolamineinduced cognitive deficits in mice," Pharmacology Biochemistry \& Behavior, vol. 104, no. 1, pp. 138-143, 2013.

[69] S. Gauthier, "Advances in the pharmacotherapy of Alzheimer's disease," Canadian Medical Association Journal, vol. 166, no. 5, pp. 616-623, 2002.

[70] M. Méndez, M. Méndez-López, L. López, M. A. Aller, J. Arias, and J. L. Arias, "Acetylcholinesterase activity in an experimental rat model of Type C hepatic encephalopathy," Acta Histochemica, vol. 113, no. 3, pp. 358-362, 2011.

[71] E. Mikiciuk-Olasik, P. Szymański, and E. Zurek, "Diagnostics and therapy of Alzheimer's disease," Indian Journal of Experimental Biology (IJEB), vol. 45, no. 4, pp. 315-325, 2007.

[72] A. Matsuo, J. Bellier, M. Nishimura, O. Yasuhara, N. Saito, and H. Kimura, "Nuclear choline acetyltransferase activates transcription of a high-affinity choline transporter," The Journal of Biological Chemistry, vol. 286, no. 7, pp. 5836-5845, 2011.

[73] M. D. Ikonomovic, E. J. Mufson, J. Wuu, E. J. Cochran, D. A. Bennett, and S. T. DeKosky, "Cholinergic plasticity in hippocampus of individuals with mild cognitive impairment: correlation with Alzheimer's neuropathology," Journal of Alzheimer's Disease, vol. 5, no. 1, pp. 39-48, 2003.

[74] C. Nyakas, I. Granic, L. G. Halmy, P. Banerjee, and P. G. M. Luiten, "The basal forebrain cholinergic system in aging and dementia. Rescuing cholinergic neurons from neurotoxic amyloid- $\beta 42$ with memantine," Behavioural Brain Research, vol. 221, no. 2, pp. 594-603, 2011.

[75] N. Tabet, D. Mantle, and M. Orrell, "Free radicals as mediators of toxicity in Alzheimer's disease: A review and hypothesis," Adverse Drug Reactions and Toxicological Reviews, vol. 19, no. 2, pp. 127-152, 2000.

[76] A. T. Eduviere, S. Umukoro, A. O. Aderibigbe, A. M. Ajayi, and F. A. Adewole, "Methyl jasmonate enhances memory performance through inhibition of oxidative stress and acetylcholinesterase activity in mice," Life Sciences, vol. 132, pp. 20-26, 2015.

[77] Y. Hu, Y. Yang, M. Zhang, M. Deng, and J.-J. Zhang, "Intermittent fasting pretreatment prevents cognitive impairment in a rat model of chronic cerebral hypoperfusion," Journal of Nutrition, vol. 147, no. 7, pp. 1437-1445, 2017.

[78] G. Vinothkumar, C. Kedharnath, S. Krishnakumar et al., "Abnormal amyloid $\beta 42$ expression and increased oxidative stress in plasma of CKD patients with cognitive dysfunction: A small scale case control study comparison with Alzheimer's disease," BBA Clinical, vol. 8, pp. 20-27, 2017.

[79] A. Prakash, B. Shur, and A. Kumar, "Naringin protects memory impairment and mitochondrial oxidative damage against aluminum-induced neurotoxicity in rats," International Journal of Neuroscience, vol. 123, no. 9, pp. 636-645, 2013.

[80] G. L. Viswanatha, H. Shylaja, and Y. Moolemath, “The beneficial role of Naringin- a citrus bioflavonoid, against oxidative stressinduced neurobehavioral disorders and cognitive dysfunction in rodents: A systematic review and meta-analysis," Biomedicine \& Pharmacotherapy, vol. 94, pp. 909-929, 2017.

[81] A. Pandey, S. Bani, P. Dutt, N. Kumar Satti, K. Avtar Suri, and G. Nabi Qazi, "Multifunctional neuroprotective effect of Withanone, a compound from Withania somnifera roots in 
alleviating cognitive dysfunction," Cytokine, vol. 102, pp. 211221, 2018.

[82] A. Ciobica, L. Hritcu, V. Artenie, and M. Padurariu, "The effects of some cholinergic drugs on cognitive processes and oxidative stress in rat," Revista Medico-Chirurgicala a Societatii De Medici Si Naturalisti Din Iasi, vol. 113, no. 3, pp. 832-837, 2009.

[83] F. Cesca, P. Baldelli, F. Valtorta, and F. Benfenati, “The synapsins: key actors of synapse function and plasticity," Progress in Neurobiology, vol. 91, no. 4, pp. 313-348, 2010.

[84] J. G. Howland and Y. T. Wang, "Synaptic plasticity in learning and memory: stress effects in the hippocampus," Progress in Brain Research, vol. 169, pp. 145-158, 2008.

[85] Y.-Z. Ling, W. Ma, L. Yu, Y. Zhang, and Q.-S. Liang, "Decreased PSD95 expression in medial prefrontal cortex (mPFC) was associated with cognitive impairment induced by sevoflurane anesthesia," Journal of Zhejiang University SCIENCE B, vol. 16, no. 9, pp. 763-771, 2015.

[86] K. Nagao, K. Nakamitsu, H. Ishida et al., "A comparison of the lipid-lowering effects of four different $n-3$ highly unsaturated fatty acids in HepG2 cells," Journal of Oleo Science, vol. 63, no. 10, pp. 979-985, 2014.

[87] N. C. Berchtold, G. Chinn, M. Chou, J. P. Kesslak, and C. W. Cotman, "Exercise primes a molecular memory for brain-derived neurotrophic factor protein induction in the rat hippocampus," Neuroscience, vol. 133, no. 3, pp. 853-861, 2005.

[88] P. Valente, S. Casagrande, T. Nieus et al., "Site-specific synapsin I phosphorylation participates in the expression of post-tetanic potentiation and its enhancement by BDNF," The Journal of Neuroscience, vol. 32, no. 17, pp. 5868-5879, 2012.

[89] B. E. Deverman and P. H. Patterson, "Cytokines and CNS development," Neuron, vol. 64, no. 1, pp. 61-78, 2009.

[90] M. Kaneko, D. Stellwagen, R. C. Malenka, and M. P. Stryker, "Tumor necrosis factor-alpha mediates one component of competitive, experience-dependent plasticity in developing visual cortex," Neuron, vol. 58, no. 5, pp. 673-680, 2008.

[91] J. G. Robinson and N. J. Stone, "Antiatherosclerotic and Antithrombotic Effects of Omega-3 Fatty Acids," American Journal of Cardiology, vol. 98, no. 4, pp. 39-49, 2006. 


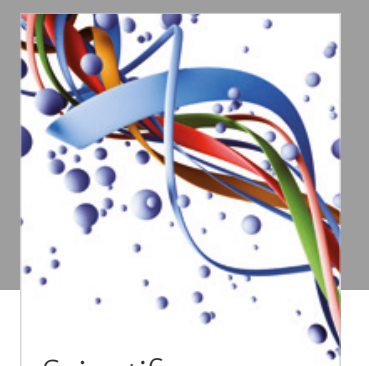

Scientifica
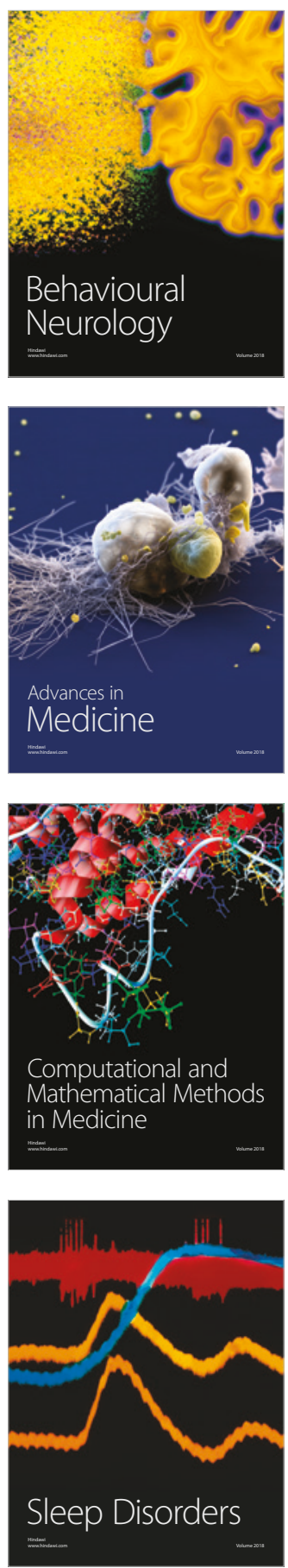

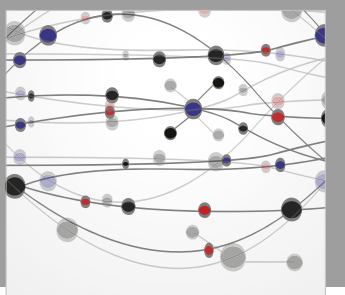

The Scientific World Journal

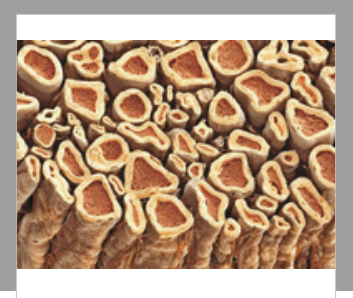

Case Reports in

Neurological Medicine

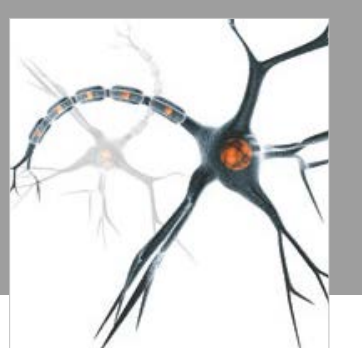

Neural Plasticity

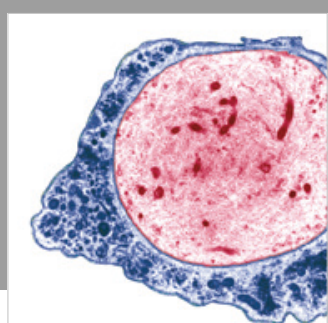

Multiple Sclerosis

International

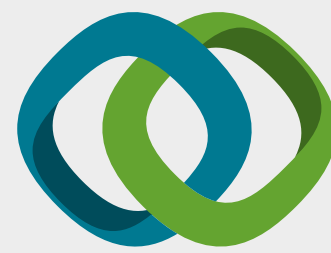

Hindawi

Submit your manuscripts at

www.hindawi.com
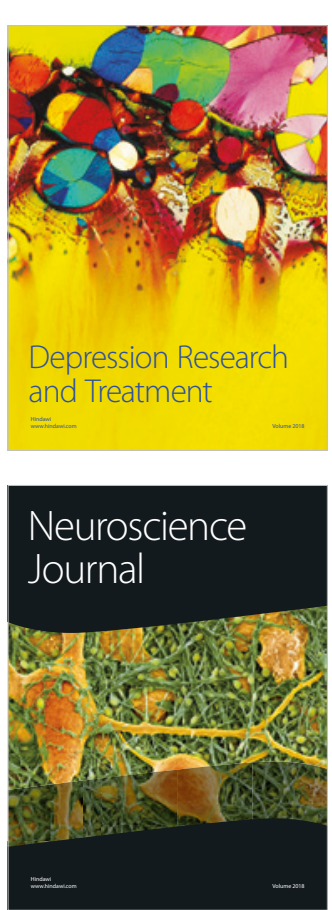

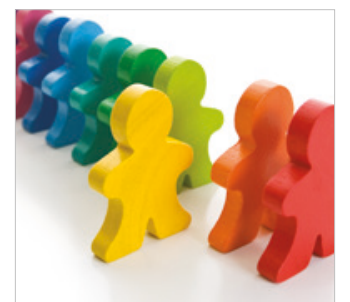

Autism

Research and Treatment
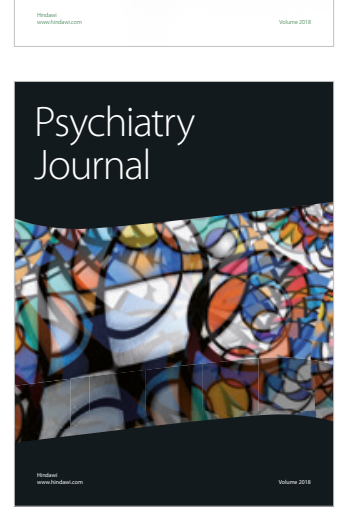
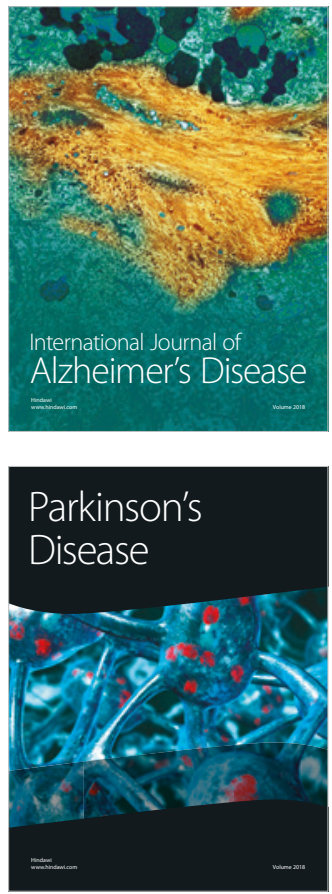
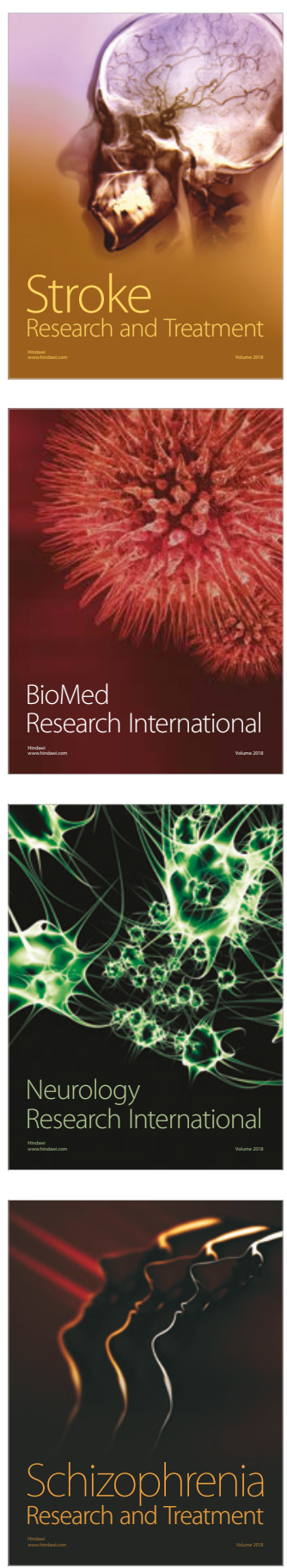